\title{
Gut Microbe-Targeted Choline Trimethylamine Lyase Inhibition Improves Obesity Via \\ Rewiring of Host Circadian Rhythms.
}

Rebecca C. Schugar ${ }^{1,2^{\star}}$, Christy M. Gliniak ${ }^{1,2^{\star}}$, Robert N. Helsley ${ }^{1,2}$, Amanda L. Brown ${ }^{1,2}$, Amy Burrows ${ }^{1,2}$, Chelsea Finney ${ }^{1,2}$, Kevin K. Fung ${ }^{1,2}$, Frederick M. Allen ${ }^{1,2}$, Daniel Ferguson ${ }^{1,2}$, Anthony D. Gromovsky ${ }^{1,2}$, Chase Neumann ${ }^{1,2}$, Amy McMillan ${ }^{1,2}$, Jennifer A. Buffa ${ }^{1,2}$, James T. Anderson ${ }^{1,2}$, Margarete Mehrabian ${ }^{5}$, Maryam Goudzari ${ }^{1,2,4}$, Belinda Willard ${ }^{1,2,4}$, Tytus D. Mak ${ }^{8}$, Andrew R. Armstrong ${ }^{7}$, Garth Swanson ${ }^{7}$, Ali Keshavarzian ${ }^{7}$, Jose Carlos Garcia-Garcia ${ }^{6}$, Zeneng Wang ${ }^{1,2}$, Aldons J. Lusis ${ }^{5}$, Stanley L. Hazen ${ }^{1,2,3}$, and J. Mark Brown ${ }^{1,2 \#}$

${ }^{1}$ Department of Cardiovascular and Metabolic Sciences, Lerner Research Institute Cleveland Clinic, Cleveland, OH 44195, USA

${ }^{2}$ Center for Microbiome and Human Health, Lerner Research Institute, Cleveland Clinic, Cleveland, OH 44195, USA

${ }^{3}$ Department of Cardiovascular Medicine, Heart and Vascular Institute, Cleveland Clinic, Cleveland, OH 44195 USA

${ }^{4}$ Proteomics and Metabolomics Core, Lerner Research Institute, Cleveland Clinic, Cleveland, $\mathrm{OH} 44195$

${ }^{5}$ Departments of Medicine, Microbiology, and Human Genetics, University of California Los Angeles, Los Angeles, CA 90095, USA

${ }^{6}$ Life Sciences Transformative Platform Technologies, Procter \& Gamble, Cincinnati, OH, USA

${ }^{7}$ Department of Internal Medicine, Division of Gastroenterology, Rush University Medical Center, Chicago, IL 60612, USA

${ }^{8}$ Mass Spectrometry Data Center, National Institute of Standards and Technology (NIST), Gaithersburg, MD 20899, USA

${ }^{*}$ Contributed equally to the work

\# = To whom correspondence should be addressed: Department of Cardiovascular and Metabolic Sciences, Cleveland Clinic, Cleveland, OH 44195, USA. Tel: 216-444-8340; Fax: 216444-9404; E-mail: brownm5@ccf.org

Running Title: TMA lyase inhibition improves obesity

Key Words: obesity, microbiome, insulin resistance, circadian 
Abbreviations used: BMAL1, aryl hydrocarbon receptor nuclear translocator like; Cry1, cryptochrome 1; CutC, choline trimethylamine lyase; CVD, cardiovascular disease; DIO, dietinduced obesity; Fmo3, flavin containing monooxygenase 3; IMC, iodomethylcholine; LPC, lysophosphatidylcholine; Per2, period 2; PC, phosphatidylcholine; PCA, principal component analysis; RevErb $\alpha$, nuclear receptor subfamily 1 group $D$ member 1; TMA, trimethylamine; TMAO, trimethylamine $\mathrm{N}$-oxide; ZT, zeitgeber.

\section{Abstract}

Obesity has repeatedly been linked to reorganization of the gut microbiome, yet to this point obesity therapeutics have been targeted exclusively toward the human host. Here we show that gut microbe-targeted inhibition of the trimethylamine $\mathrm{N}$-oxide (TMAO) pathway protects mice against the metabolic disturbances associated with diet-induced obesity (DIO) or leptin deficiency (ob/ob). Small molecule inhibition of the gut microbial enzyme choline TMA-lyase (CutC) does not reduce food intake, but is instead associated with beneficial remodeling of the gut microbiome, improvement in glucose tolerance, and enhanced energy expenditure. We also show that CutC inhibition is associated with reorganization of host circadian control of both phosphatidylcholine and energy metabolism. This study underscores the relationship between microbe and host metabolism, and provides evidence that gut microbe-derived trimethylamine (TMA) is a key regulator of the host circadian clock. This work also demonstrates that gut microbe-targeted enzyme inhibitors have untapped potential as anti-obesity therapeutics.

\section{Introduction}

There is a growing body of evidence that microbes residing in the human intestine represent a critical environmental factor that influences virtually all aspects of human health and disease ${ }^{5,6}$. In fact, our gut microbiome plays a central role in vital processes such as energy harvest from 
our diet, entraining our immune system during early life, xenobiotic metabolism, and the production of a diverse array of small molecule metabolites that are essential to human life ${ }^{5,6}$. Although the microbiome field has uncovered many correlative relationships with human health and disease, there are few examples whereby alterations in the gut microbiome have been causally linked. One of the earliest and reproducible causal relationships established between gut microbes and human disease was is the link between alterations in gut microbial phyla (Bacteroidetes and Firmicutes) and obesity susceptibility, and the microbial transplantation studies revealing obesity susceptibility as a transmissible trait ${ }^{1-4}$. Specifically, obese mice harbor gut microbial communities with enhanced capacity to harvest energy from indigestible carbohydrates $^{3}$, and transplantation of either cecal contents or feces from either obese mice or humans is sufficient to promote obesity and related insulin resistance in germ-free mouse recipients $^{1-4}$. There is also accumulating evidence that antibiotic exposure in early life can predispose children to become overweight or obese later in life ${ }^{7}$, and antibiotic treatment in mice prior to weaning increases obesity and related insulin resistance in adulthood ${ }^{8}$. In fact, nearly all diseases where obesity is a predisposing comorbidity (diabetes, liver disease, cardiovascular disease, hypertension, chronic kidney disease, and diverse cancers) have been shown to have a clear gut microbiome link ${ }^{9-14}$.

Although there is now ample evidence that gut microbes play a contributory role in the development of obesity and related metabolic disorders, obesity-targeted drug discovery to this point has focused solely on targets encoded by the human genome. Our knowledge is rapidly expanding in regards to what types of microbes are associated with obesity and related disorders, including the repertoire of microbe-associated molecule patterns (MAMPs) they harbor and the vast array of metabolites that they produce. However, there are very few examples of where this information has been leveraged into clinically relevant therapeutics strategies. The microbiome-targeted therapeutic field has primarily focused on either pre-biotic 
or pro-biotic approaches, yet thus far these community restructuring approaches have resulted in very modest or non-significant effects in obesity-related disorders in human studies ${ }^{15-19}$. As an alternative microbiome-targeted approach, we and others have begun developing non-lethal selective small molecule inhibitors of bacterial enzymes with the hopes of reducing levels of disease-associated microbial metabolites ${ }^{20-24}$. In fact, we have recently shown that small molecule inhibition of the gut microbial transformation of choline into trimethylamine (TMA), the initial and rate-limiting step in TMAO generation ${ }^{25}$, can significantly reduce atherosclerosis, thrombosis, and adverse ventricular and kidney remodeling in mice $20-23$. In a day where host genetics/genomics approaches dominate, the discovery that the gut microbial TMAO pathway plays a contributory role in cardiovascular disease (CVD) risk highlights that the interplay between our diet and gut microbial enzymology play an extremely important role in modulating human disease susceptibility ${ }^{25}$. In fact, since 2011 the gut microbe-associated TMAO pathway has been associated with many human diseases associated with obesity including atherosclerosis ${ }^{25,26}$, thrombosis ${ }^{27,28}$, chronic kidney disease 29,30 , heart failure ${ }^{31,32}$, cancer ${ }^{33,34}$, and diabetes ${ }^{35,36}$. Given the fact that obesity is a comorbidity in all of these human diseases, here we set out to determine whether selective small molecule inhibition of the gut microbial choline transformation into TMA, a metabolic activity catalyzed by the microbial choline TMA lyase CutC $^{37}$, can protect against metabolic disturbance in preclinical mouse models of obesity.

\section{Microbial choline TMA lyase inhibition protects mice from obesity development}

To assess whether small molecule inhibition of gut microbial TMA production can protect mice from obesity, we treated mice with the non-lethal mechanism-based bacterial choline TMA lyase inhibitor iodomethylcholine $(\mathrm{IMC})^{20}$. This small molecule inhibitor exhibits potent in vivo inhibition of the gut microbial choline TMA lyase enzyme CutC, lowering host plasma TMAO levels $>90 \% \%^{20,22,23}$. Designed as a suicide substrate mechanism-based inhibitor, past studies reveal 
the vast majority of IMC is retained in bacteria and excreted in the feces with limited systemic exposure of the polar drug in the host ${ }^{20}$. When administered in a high fat diet (HFD), IMC effectively reduces levels of both the primary product of CutC TMA as well as the host liverderived co-metabolite TMAO (Figure 1a,b). IMC was effective in blunting diet-induced obesity (DIO) (Figure 1c), without altering food intake (Fig. 1d). DIO mice treated with IMC also showed improvements in glucose tolerance (Figure 1e), and exhibited marked reductions in plasma insulin levels in fed mice but not under fasting conditions (Figure 1f). Next, we administered IMC to hyperphagic leptin-deficient (ob/ob) mice in a chow-based diet (Figure 1g-I). As expected, IMC effectively lowered both TMA and TMAO levels in ob/ob mice (Figure 1g,h). Quite unexpectedly, IMC dramatically protected ob/ob mice from body weight gain, where some of the drug treated mice were 15-20 grams lighter than control ob/ob mice (Figure 1i). Notably, IMCdriven protection from obesity was not associated with decreased food intake, which was paradoxically higher overall in IMC-treated mice (Figure 1j). Instead, IMC treatment promoted large increases in energy expenditure through both the light and dark cycles (Figure 1I, Figure 1 - figure supplement 1) Although IMC effectively reduced body weight and adiposity in ob/ob mice, this was not associated with improvements in glucose tolerance (Figure 1k). Given these striking results in DIO and ob/ob obesity progression models, we next wanted to determine if IMC could likewise provide obesity protection in mice with established obesity (i.e. a treatment regimen). To test this we fed C57BL/6J mice HFD for 6 weeks to allow for mice to reach obese conditions (body weights ranging from 35-40 grams) and then initiated IMC treatment. Although IMC did not promote weight loss, it did effectively reduce the trajectory of weight gain and improved glucose tolerance in this obesity treatment model (Figure 1 - figure supplement 1). During the early phases of this obesity treatment study, IMC-treated mice again exhibited unexpected increased food intake compared to controls (Figure 1 - figure supplement 1). To more comprehensively understand the global effects of choline TMA lyase inhibition on white 
adipose tissue gene expression, we performed unbiased RNA sequencing (Figure 1 - figure supplement 1). Principal coordinate analysis (PCA) of RNA expression profiles showed clear separation of adipose gene expression between HFD control versus HFD with IMC (Figure 1 figure supplement 1). The most differentially expressed genes altered by IMC were enriched in common pathways of adipogenesis, fatty acid metabolism, inflammation, and cytokine signaling (Figure 1 - figure supplement 1). Collectively, these data demonstrate that gut microbe-targeted choline TMA lyase inhibition can protect mice from obesity and selectively reorganize host adipose tissue gene expression.

\section{Choline TMA lyase inhibition promotes beneficial remodeling of the gut microbiome}

One theoretical advantage of non-lethal microbe-targeted choline TMA lyase inhibitors, compared to antibiotic therapies, is that microbiome restructuring effects of the drug are expected to be primarily target the species that rely on choline as a carbon or nitrogen source. Therefore, we next examined whether IMC treatment was associated with alterations in choline utilizers and other members of the gut microbiome community that may contribute to improvement in obesity-related metabolic disturbances. IMC treatment in HFD-fed mice resulted in significant restructuring of the cecal microbiome at every taxonomic level (Figure 2a-e). PCA of microbial taxa revealed distinct clusters, indicating that IMC promoted restructuring of the cecal microbiome (Figure 2a). At the phylum level, HFD-fed IMC-treated mice had large increases in Verrucomicrobia and Bacteroidetes, and significant reductions in Firmicutes (Figure $2 \mathrm{~b}, \mathrm{c})$. It is important to note that the ratio of Firmicutes to Bacteroidetes, which has been repeatedly associated with obesity in both humans and mice ${ }^{1-4}$, is significantly reduced by IMC treatment of the HFD-fed mice (Figure 2c). Performance of linear discriminant analysis coupled with effect size measurements (LEfSe analysis) revealed that IMC promoted significant reductions in the proportions of the taxa Desulfovibrionaceae, Butyrovibrio, Bilophia, 
Peptococcaceae, and Dorea, and increases in Odoribacter, Roseburia, and several members of the Clostridiaceae family (Figure 2d). We also examined whether the proportions of cecal genera were significantly correlated with plasma TMA, body weight, and glucose tolerance across in DIO mice, and found that increases in Akkermansia and reductions in Bilophila were significantly correlated with plasma TMA level, and similar yet non-significant trends were found with body weight and glucose tolerance (Figure 2e). Similar to HFD-fed mice (Figure 2a-e), IMC also significantly altered the cecal microbiome in ob/ob mice (Figure 2f-2i). In ob/ob mice, IMC significantly reduced the proportions of Adlercreutzia, Rikenellaceae, Peptococcaceae, and Clostridiales, while increasing levels of Molicutes, Allobaculum, Peptostreptococcaceae, and Akkermansia (Figure 2g-h). Correlation analysis in ob/ob mice revealed that IMC-induced alterations in Akkermansia, Clostridiales, and Allobaculum were significantly associated with circulating TMA and body weight (Figure 2i). Notably, in both the ob/ob and DIO models, the large increase in the proportions of Verrucomicrobia phylum seen with IMC are largely explained by increased level of Akkermansia mucinophila, which has been pursued as a therapeutic probiotic strain for diabetes amelioration in other studies ${ }^{38-40}$, and is correlated to TMA and body weight phenotypes in the current study. Collectively, these data demonstrate that inhibition of gut microbial choline to TMA transformation with a selective non-lethal small molecule inhibitor promotes beneficial restructuring of the gut microbiome that may contribute in part to improvements in energy metabolism and obesity observed in the host.

\section{The gut microbial TMAO pathway regulates host circadian rhythms}

Although there is clear evidence that TMAO can directly impact cell signaling in macrophages ${ }^{41}$, endothelial cells ${ }^{41}$, and platelets ${ }^{27}$, it is still incompletely understood how the TMAO pathway is mechanistically linked to all of these common obesity-related diseases. Because of the recent appreciation of the gut microbiome as a coordinator of host metabolism and obesity-related 
phenotypes $^{40-43}$, we hypothesized that the metaorganismal TMAO pathway regulates host circadian rhythms to impact obesity and its associated cardiometabolic complications. The data that led us to this possibility includes: (1) misalignment of the host circadian clock is associated with the same human diseases that the TMAO pathway has been linked to ${ }^{40-43},(2)$ the gut microbiome oscillates in a circadian manner and this is highly gender specific (similar to the TMAO pathway $)^{40-42}$, and (3) disruption of the host circadian core clock machinery promotes gut microbial "dysbiosis" and alters circulating levels of gut-derived metabolites ${ }^{40-43}$. Given these clear overlaps, we investigated whether different substrates and regulatory genes within the TMAO pathway exhibited circadian rhythmicity. Indeed, we found that several nodes within the gut microbial TMAO pathway showed strong diurnal oscillations in mice (Figure 3a). Plasma levels of both choline, carnitine, and TMA were relatively low during the light cycle, and peak at the beginning of the dark cycle when mice are most active and consuming food (Figure 3a). However, TMAO showed more modest oscillatory behavior (Figure $3 a$ and Figure 3 - figure supplement 1 ). We also found that the hepatic expression of flavin-containing monooxgygenase 3 (Fmo3) is quite low during the day, but increases 6-fold during the early dark cycle (Figure 3a). However, this pattern for Fmo3 expression is opposite in white adipose tissue (Figure 4 - figure supplement 1). Unexpectedly, we identified a tissue-specific circadian rhythm in the host TMA receptor (trace amine-associated receptor 5; Taar5) (Figure 3a). It is important to note that Taar5 oscillation specifically occurs in skeletal (Figure 3a; Figure 3 - figure supplement; Figure 4 - figure supplement 1) and cardiac muscle (Figure 4 - figure supplement 1), and shows no oscillatory behavior in the olfactory bulb (Figure 4 - figure supplement 1), where it is important for sensing the "fishy odor" smell of TMA ${ }^{44,45 .}$ Collectively, these data suggest that the gut microbial TMAO pathway dynamically oscillates in a circadian manner.

\section{Gut microbe-targeted inhibition of TMA production rewires host-microbe metabolic}




\section{crosstalk}

To determine whether TMA or TMAO could potentially serve as a gut microbe-derived signal to entrain the host circadian clock we treated a set of mice with IMC acutely (7 days) and examined effects on the core clock machinery as well as clock-mediated regulation of host metabolism. In this circadian study, IMC treatment effectively lowered circulating TMA and TMAO at every time point, yet there was still a highly rhythmic low level of plasma TMA and TMAO observed, possibly originating from microbial metabolism of non-choline (i.e. carnitine, $\gamma$-butyrobetaine, betaine, trimethyllysine) nutrient sources via microbial enzymes that are not inhibited by IMC ${ }^{46-48}$ (Figure 3 - figure supplement 1). Unexpectedly, Provision of IMC altered the expression of core clock transcription factors in a highly tissue-specific manner. In the liver, microbial choline TMA lyase inhibition modestly increased the peak amplitude of the core clock transcription factors Bmal1 and Rev-erba, yet blunted the rhythmic expression of Cry1 (Figure 3b). In white adipose tissue, choline TMA lyase inhibition blunted the peak amplitude of Bmal1 (Figure 3b). However, in skeletal muscle we found the most striking differences in the core clock machinery where IMC-treated mice showed marked increases at all time points for Rev-erba and a near complete phase shift for Cry1 (Figure 3b; Figure 3 - figure supplement 1). Prior studies have confirmed that IMC is poorly absorbed in the host and selectively accumulates in gut microbiota ${ }^{20,22}$. It is tempting to speculate that the circadian oscillations in skeletal muscle are so drug responsive due in part to the fact that the TMA receptor Taar5 oscillates there (Figure 3a; Figure 3 - figure supplement 1 ) to sense TMA and is not a "direct" result of IMC per se, but rather IMC effect on gut microbiota production of TMA. It is important to note that IMC does not alter the expression of core clock genes in area of the central nervous system like the olfactory bulb where Taar5 is abundantly expressed throughout the day and does not oscillate diurnally.

Recent literature has demonstrated that the composition of the gut microbiome is also under circadian regulation ${ }^{40-42}$. These reports show that even at the phylum level, bacterial communities 
oscillate in a highly circadian manner, and gut microbe community oscillation can impact host metabolic rhythms that are linked to human disease ${ }^{40-42}$. However, there is almost nothing known regarding what regulates this diurnal rhythm in gut microbial communities. Here we demonstrate that microbial production of TMA, as modulated by the choline TMA lyase inhibitor IMC, has striking effects on circadian oscillations in gut microbial communities (Figure 2 - figure supplement 1). PCA analyses revealed distinct group clusters, indicating that IMC promoted restructuring of the cecal microbial community composition in a circadian manner (Figure 2 - figure supplement 1). At the phylum level, IMC treatment results in large relative increases in Bacteroidetes, while lowering the overall levels of Firmicutes at all time points (Figure 2 - figure supplement 1). Interestingly, IMC treatment promoted a significant increase in the abundance of Akkermansia mucinophila, proportions of which have been reported to be inversely correlated with body weight and insulin sensitivity in numerous studies in both rodents and humans ${ }^{38-40}$. In addition, IMC treatment increased the level of S24-7 Bacteroidetes family members, which have recently been reported to be depleted in several mouse models of obesity-related metabolic disturbance ${ }^{49-51}$. Collectively, these data demonstrate that gut microbial production of TMA may be an important cue to impact the diurnal oscillations in gut microbial community structure and metabolic function in the host.

In an attempt to understand how choline TMA lyase inhibition altered host metabolic processes in an unbiased way, we performed untargeted plasma metabolomics across a 24hour period in IMC-treated mice (Figure 4). This large circadian metabolomics dataset was initially evaluated for any large-scale trends utilizing Selective Paired Ion Contrast Analysis $(\text { SPICA })^{52}$ to identify key zeitgeber $(\mathrm{ZT})$ time points where IMC elicited the most significant alterations in the plasma metabolome (Figure 4a). Although there were clear IMC-induced metabolomic alterations at every time point, the most significant alterations were seen during the ZT10 to ZT14 transition, which coincides with the light to dark transition when mice typically 
begin to forage for food. Focusing on the ZT14 time point, we observed a large number of differentially abundant metabolites in the IMC-treated group that were in common pathways of lipid and lipoprotein metabolism, phase II conjugation, and other various macronutrient metabolic pathways (Figure 4b,c). In particular, we found that choline TMA lyase inhibition was associated with alterations in diurnal rhythms of phosphatidylcholine (PC) co-metabolites (Figure 4d). In fact, gut microbial TMA lyase inhibition caused near complete circadian phase shifts for two PC species (16:0/22:6 and 18:1/18:1), and two saturated species (16:0 and 18:0) of lysophosphatidylcholine (LPC), which showed robust oscillatory behavior distinct from control mice (Figure 4d). Furthermore, we found that the oscillatory behavior of genes involved in PC synthesis including choline kinase $\alpha(\mathrm{CK \alpha})$ and phosphatidylethanolamine methyltransferase (PEMT) were significantly altered in TMA lyase inhibited mice (Figure 3 - figure supplement 1 ). It is important to note that IMC did not alter key plasma hormones (corticosterone or insulin) or lipids (non-esterified fatty acids) that are known to have strong effects on phospholipid metabolism themselves (Figure 4 - figure supplement 1). Collectively, these data suggest that gut microbial TMA production from the choline TMA lyase CutC is a critical determinant of host circadian rhythms in PC homeostasis.

\section{Discussion}

Although obesity drug discovery has historically targeted pathways in the human host, a fertile period in biomedical research lies ahead where we instead we target the microorganisms that live within us to improve obesity and related disorders. This paradigm shift in drug discovery is needed in light of the clear and reproducible associations between the gut microbiome and almost every human disease. Now we are faced with both the challenge and opportunity to test whether microbe-targeted therapeutic strategies can improve health in the human metaorganism without negatively impacting the symbiotic relationships that have co-evolved. Although 
traditional microbiome manipulating approaches such as antibiotics, prebiotics, probiotics, and fecal microbial transplantation have shown their own unique strengths and weaknesses, each of these present unique challenges particularly for use in chronic diseases such as obesity. As we move toward selective nonlethal small molecule therapeutics, the hope is to have exquisite target selectivity and limited systemic drug exposure given the targets are microbial in nature. This natural progression parallels the paradigm shifts in oncology which have transitioned from broadly cytotoxic chemotherapies to target-selective small molecule and biologics-based therapeutics. Here we provide the first evidence that a selective nonlethal inhibitor to the microbial choline TMA lyase CutC can have beneficial effects on host energy metabolism, adiposity, and insulin sensitivity. The current study demonstrates that gut microbe-targeted suppression of choline to TMA generation: (1) protects the host from diet-induced obesity and glucose intolerance, (2) protects ob/ob mice from obesity, (3) increases energy expenditure and alters the expression of lipid metabolic genes in white adipose tissue, and (4) beneficially reorganizes gut microbial communities to improve the obesity related Firmicutes to Bacteroidetes ratio and increase abundance of Akkermansia mucinophila. This study also reveals that nodes within the gut microbial TMAO pathway exhibit circadian oscillatory behavior, and that inhibition of microbial choline to TMA transformation is associated with alterations in: (1) the expression of core clock machinery in metabolic tissues; (2) the diurnal rhythmic behavior of gut microbial communities; and (3) circadian oscillation in choline-containing phospholipids. Collectively, our findings reinforce the notion that the gut microbial TMAO pathway is a strong candidate for therapeutic intervention across a spectrum of obesity-related diseases, and have uncovered metaorganismal crosstalk between gut microbe-derived TMA and circadian metabolic oscillations in mice. Interestingly, during the preparation of this manuscript a recent meta-analysis of clinical studies demonstrated that circulating TMAO levels are dosedependently associated with obesity in humans ${ }^{53}$, highlighting the potential translation of this 
work.

As drug discovery advances in the area of small molecule nonlethal bacterial enzyme inhibitors it is key to understand how these drugs impact microbial ecology in the gut and other microenvironments. As we have previously reported $20,22,23$, IMC treatment does promote a significant remodeling of the cecal microbiome in mice. These IMC-induced alterations occur as early as one week (Figure 2 - figure supplement 1) and persist even after twenty weeks (Figure 2). It is important to note that the observed IMC-induced alterations in the gut microbiome are generally expected to be beneficial. For instance, IMC lowers the ratio of Firmicutes to Bacteroidetes (Figure 2c), which is known to be elevated in obese humans and rodents ${ }^{1-4}$. Of the taxa that were significantly correlated with circulating TMA levels (Akkermansia, Bilophila, Clostridiales, and Allobaculum), only Akkermansia was likewise correlated with body weight in ob/ob mice (Figure 2i). It is important to note that previous studies have shown beneficial effects of Akkermansia mucinophila in obesity and diabetes, which has prompted its development as a probiotic $^{36-38}$. In fact supplementation with Akkermansia mucinophila can improve glucose tolerance in obese mice ${ }^{38-40}$. It is important to note that increases in Akkermansia mucinophila has been consistently seen in mice treated with TMA lyase inhibitor ${ }^{20,22,23}$, suggesting that small molecule inhibition of gut microbial choline TMA lyase inhibitors may be an alternative means to enrich gut microbiomes with Akkermansia mucinophila in addition to developing probiotic approaches. As small molecule bacterial enzymes inhibitors are developed it will be extremely important to understand their effects on microbial ecology, and it is expected that some of the beneficial effects of these drugs will indeed originate from the restructuring of gut microbiome communities. In fact, this is not an uncommon mechanism by which host targeted drugs impact human health. A recent study showed that nearly a quarter of commonly used host-targeted drugs have microbiome-altering properties ${ }^{54}$, and in the context of diabetes therapeutics it is important to note metformin's anti-diabetic effects are partially mediated by the drug's 
microbiome altering properties ${ }^{55}$. Given the strong association between gut microbiome and obesity and diabetes ${ }^{1-4}$, it will likely be advantageous to find therapeutics that beneficially remodel the gut microbiome as well as engage either their microbe or host target of interest.

One burgeoning area of microbe-host cross talk relevant to human disease is the intersection between gut microbes and host circadian rhythms ${ }^{40-43}$. It is well appreciated that a transcriptional-translational feedback loop (TTFL) exists in mammalian cells to orchestrate an approximately 24-hour oscillatory rhythm in the expression of thousands of genes ${ }^{40-43}$. The mammalian clock is coordinated by core transcription factors CLOCK and BMAL1, which peak during light phases, and crytochromes (CRYs) and period genes (PERs), which are most active during dark phases. Under normal conditions, the clock regulated TTFL maintains cell autonomous homeostatic responses to environmental "zeitgebers" including light, food, xenobiotics, and exercise ${ }^{40-43}$. However, disruption of normal circadian rhythms induced by abnormal light exposure, sleep-activity, or eating patterns has been associated with the development of many human diseases including obesity, diabetes, CVD, kidney disease, cancer, and neurological disease ${ }^{40-43}$. Therefore, "chronotherapies" or therapies that prevent circadian disruption hold promise across a wide spectrum of diseases. Interestingly, it has been discovered that circadian disruption is associated with a marked reorganization of gut microbial communities, and microbial abundance in the gut exhibits circadian rhythmicity ${ }^{40-43}$. However, whether gut microbial metabolites contribute to circadian disruption is not well understood. Here we show that multiple nodes with the metaorganismal TMAO pathway (choline, TMA, Fmo3, and Taar5) oscillate in a circadian manner, and that inhibition of the choline TMA lyase CutC/D rewires the host circadian clock itself, as well as circadian rhythms in the cecal microbiome, as well as clock-driven reorganization of host lipid metabolic processes. The impact of gut microbial choline TMA lyase inhibition on the expression of core clock genes is modes in metabolic tissues such as the liver or white adipose tissue (Figure 3b; Figure 3 - figure supplement 1). However, 
in skeletal muscle, where the host TMA receptor Taar5 exhibits oscillatory behavior (Figure 3a), IMC treatment elicits profound alterations in the expression of Nr1d1 (RevErb $\alpha)$ and cryptochrome 1 (Cry1). The oscillatory behavior of the TMA receptor Taar5 is specific to both skeletal and cardiac muscle, and is not seen in the olfactory bulb where Taar5 is essential to sense the fish-like odor of TMA ${ }^{44,45}$. A recent report demonstrated that TMAO, but not TMA, can bind to and activate the endoplasmic reticulum stress (ER stress)-related kinase PERK (EIF2AK3), and that TMAO binding to PERK regulates the expression of the forkhead transcription factor FoxO156. It is interesting to note that PERK was recently shown to regulate circadian rhythms that support sleep/wake patterns and cancer growth ${ }^{57,58}$. We also observed tissue-specific alterations in the circadian expression of FoxO1 with IMC treatment (Figure 3 figure supplement 1; Figure 4 - figure supplement 1). However, additional studies are needed to understand whether the TMAO-PERK-FOXO1 signaling axis is mechanistically linked to oscillatory behavior in metabolism or associated metabolic disease. It is interesting to note that several independent metabolomics studies have found that circulating levels of TMAO exhibit circadian oscillatory behavior ${ }^{59-61}$. In a similar manner, the expression of flavin-containing monooxygenase enzymes which convert TMA to TMAO are also under direct circadian clock transcriptional regulation ${ }^{62-64}$. Collectively, these emerging data suggest that gut microbederived TMA and potentially its metabolic co-metabolite TMAO may be underappreciated microbe-derived signals that impact host circadian rhythms in metabolism. Further studies are now warranted to test whether TMA lyase inhibitors of the host liver TMAO-producing enzyme FMO3 can act as chronotherapies to improve circadian misalignment-associated diseases.

The metaorganismal TMAO pathway represents only one of many microbial metabolic circuits that have been associated with human disease. In fact, many microbe-associated metabolites such as short chain fatty acids, secondary bile acids, phenolic acids, polyamines, among others have been associated with many human diseases ${ }^{5,11}$. In a circadian and/or meal- 
related manner gut microbes produce a diverse array of metabolites that reach micromolar to millimolar concentrations in the blood, making the collective gut microbiome an active endocrine $\operatorname{organ}^{5}$. Small molecule metabolites are well known to be mediators of signaling interactions in the host, and this work provides evidence that diet-microbe-host metabolic interplay that can shape normal diurnal rhythms in metabolism and obesity susceptibility in mice. Our work, that of many others, demonstrates that there is clear evidence of bi-directional crosstalk between the gut microbial endocrine organ and host metabolism. As drug discovery advances it will be important to move beyond targets based solely in the human host. This work highlights that nonlethal gut microbe-targeted enzyme inhibitors can serve as effective anti-obesity therapeutics in preclinical animal models, and provides proof of concept that this may be a generalizable approach to target metaorganismal crosstalk in other disease contexts. In fact, selective mechanism-based inhibition of bacterial enzymes has the advantage over host targeting given that small molecules can be designed to avoid systemic absorption and exposure, thereby minimizing potential host off target effects. As shown here with the gut microbial TMAO pathway, it is easy to envision that other microbe-host interactions are mechanistically linked to host disease pathogenesis, serving as the basis for the rational design of microbe-targeted therapeutics that improve human health.

\section{METHODS}

\section{Mice and Experimental Diets}

C57BI6/J and ob/ob mice were purchased from The Jackson Laboratory (Bar Harbor, ME) and treated with a gut microbe-targeted small molecule inhibitor of choline TMA lyase called iodomethylcholine $(\mathrm{IMC})^{20}$. For high fat diet feeding experiments, C57BI6/J were maintained on $60 \%$ high fat diet alone or with the addition of $0.06 \% \mathrm{w} / \mathrm{w}$ IMC (D12942 and custom diet 
D15102401, respectively, Research Diets, Inc.). For ob/ob and circadian studies, mice were maintained on a standardized control chow diet (TD:130104, Teklad) with or without supplemental with IMC $(0.06 \% \mathrm{w} / \mathrm{w})$. Body weight was measured weekly. Food intake was assessed by weighing the food consumed weekly divided by the number of mice in each cage and normalized to the average body weight. For circadian rhythm studies, 9-week old C57BI6/J male mice were adapted for 2 weeks on a standardized minimal choline chow (Envigo diet \# TD.130104) with a strict $12 \mathrm{~h}: 12 \mathrm{~h}$ light:dark cycle after shipment. Mice were then maintained on standardized chow (Envigo diet \# TD.130104) or the same diet supplemented with $0.06 \%$ IMC (Envigo diet \# 150813). After 7 days, plasma and tissue collection were performed every 4 hours over a 24-hour period. All dark cycle necropsies were performed under red light conditions. In the obesity treatment study paradigm C57BI6/J mice fed a high fat diet (Research Diets \# D12942) for 6 weeks to establish obesity (body weight > 35 grams), and after 6 weeks of dietinduced obesity mice were continued on HFD alone (Research Diets \# D12942) or the same HFD containing IMC for another 10 weeks to test whether IMC can improve obesity-related phenotypes. For all studies plasma was collected by cardiac puncture, and liver, WAT, and skeletal muscle was collected, flash frozen, and stored at $-80^{\circ} \mathrm{C}$ until the time of analysis. All mice were maintained in an Association for the Assessment and Accreditation of Laboratory Animal Care, International-approved animal facility, and all experimental protocols were approved by the Institutional Animal Care and use Committee of the Cleveland Clinic.

\section{Synthesis of lodomethylcholine (IMC) lodide}

lodomethylcholine iodide was prepared using a previously-reported method using 2dimethylethanolamine and diiodomethane as reactants in acetonitrile followed by recrystallization from dry ethanol ${ }^{66} .{ }^{1} \mathrm{H}$ - and ${ }^{13} \mathrm{C}-\mathrm{NMRs}$ of IMC were both consistent with that in 
the reported literature ${ }^{66}$, as well as consistent based on proton and carbon chemical shift assignments indicated below. High resolution MS corroborated the expected cation mass and provided further evidence of structural identity.

${ }^{1} \mathrm{H}-\mathrm{NMR}\left(600 \mathrm{MHz}, \mathrm{D}_{2} \mathrm{O}\right): \delta 5.13\left(\mathrm{~s}, 2 \mathrm{H},-\mathrm{N}-\underline{\mathrm{C}}_{2}-\mathrm{I}\right), 3.90\left(\mathrm{t}, \mathrm{J}=4.8 \mathrm{~Hz}, 2 \mathrm{H},-\mathrm{CH}_{2}-\underline{\mathrm{H}}_{2}-\mathrm{OH}\right), 3.52$ $\left(\mathrm{t}, \mathrm{J}=4.8 \mathrm{~Hz}, 2 \mathrm{H},-\mathrm{N}-\underline{\mathrm{CH}}_{2}-\mathrm{CH}_{2}-\right), 3.16\left(\mathrm{~s}, 6 \mathrm{H},-\mathrm{N}\left(\mathrm{CH}_{3}\right)_{2}\right)$;

${ }^{13} \mathrm{C}-\mathrm{NMR}\left(150 \mathrm{MHz}, \mathrm{D}_{2} \mathrm{O}\right): \delta 65.7\left(-\mathrm{CH}_{2}-\underline{\mathrm{C}} \mathrm{H}_{2}-\mathrm{OH}\right), 55.4\left(-\mathrm{N}-\underline{\mathrm{CH}_{2}}-\mathrm{CH}_{2}-\right), 52.4\left(-\mathrm{N}\left(\underline{\mathrm{C}} \mathrm{H}_{3}\right)_{2}\right), 32.3$ $\left(-\mathrm{N}-\underline{\mathrm{C}} \mathrm{H}_{2}-\mathrm{I}\right)$

HRMS (ESI/TOF): m/z (M+) calculated for $\mathrm{C}_{5} \mathrm{H}_{13} \mathrm{INO}, 230.0036$; found, 230.0033 .

\section{Measurement of Plasma Trimethylamine (TMA) and Trimethylamine-N-oxide (TMAO)}

Stable isotope dilution high performance liquid chromatography with on-line tandem mass spectrometry (LC-MS/MS) was used for quantification of levels of TMAO, TMA, choline, carnitine, and $\gamma$-butyrobetaine in plasma, as previously described ${ }^{67}$. Their dg(methyl)isotopologues were used as internal standards. LC-MS/MS analyses were performed on a Shimadzu 8050 triple quadrupole mass spectrometer. IMC and d2-IMC, along with other metabolites, were monitored using multiple reaction monitoring of precursor and characteristic product ions as follows: $\mathrm{m} / \mathrm{z} 230.0 \rightarrow 58.0$ for IMC; m/z $232.0 \rightarrow 60.1$ for d2-IMC; m/z $76.0 \rightarrow$ 58.1 for TMAO; m/z $85.0 \rightarrow 66.2$ for dg-TMAO; m/z $60.2 \rightarrow 44.2$ for TMA; m/z $69.0 \rightarrow 49.1$ for d9-TMA; m/z $104.0 \rightarrow 60.1$ for choline; m/z $113.1 \rightarrow 69.2$ for d9-choline; $\mathrm{m} / \mathrm{z} 118.0 \rightarrow 58.1$ for betaine; $\mathrm{m} / \mathrm{z} 127.0 \rightarrow 66.2$ for dg-betaine.

\section{Analysis of Gene Expression in Mouse Tissues}


RNA was isolated via the RNAeasy lipid tissue mini kit (Qiagen) from multiple tissues. RNA samples were checked for quality and quantity using the Bio-analyzer (Agilent). RNA-SEQ libraries were generated using the Illumina mRNA TruSEQ Directional library kit and sequenced using an Illumina HiSEQ4000 (both according to the Manufacturer's instructions). RNA sequencing was performed by the University of Chicago Genomics Facility. Raw sequence files will be deposited in the Sequence Read Archive before publication (SRA). Paired-ended 1050 bp reads were trimmed with Trim Galore (v.0.3.3, http://www.bioinformatics. babraham.ac.uk/projects/trim_galore) and controlled for quality with FastQC (v0.11.3, http://www. bioinformatics.bbsrc.ac.uk/projects/fastqc) before alignment to the Mus musculus genome (Mm10 using UCSC transcript annotations downloaded July 2016). Reads were aligned using the STAR alignerSTAR in single-pass mode (v.2.5.2a_modified, https://github.com/alexdobin/STAR $)^{68}$ with standard parameters but specifying '-alignlntronMax 100000 -quantMode GeneCounts'. Overall alignment ranged from 88-99\% with 61-75\% mapping uniquely. Transcripts with fewer than one mapped read per million (MMR) in all samples were filtered out before differential expression (DE) analysis. The filtering step removed 12,692/24,411 transcripts (52\%). Raw counts were loaded into R (http://www.R-project.org/) (R Development Core Team, 2015) and edgeR ${ }^{69}$ was used to perform upper quantile, betweenlane normalization, and DE analysis. Values generated with the cpm function of edgeR, including library size normalization and log2 conversion, were used in figures. Heat maps were generated of top 50 differentially expressed transcripts using pheatmap ${ }^{70}$. Reactome-based pathway analysis was performed using an open-sourced $\mathrm{R}$ package: ReactomePA ${ }^{71}$. For real time quantitative PCR (qPCR) analyses $\sim 20 \mathrm{mg}$ of snap-frozen liver tissue was homogenized in the 1-mL TRIzol reagent (Thermo Fisher Scientific, Cat. No. 15596018). Furthermore, $200 \mathrm{~mL}$ of chloroform were added and samples were spun down at 13,000 revolutions/min for 5 min. Upper clear layer were passed through the RNeasy Mini Spin Columns (Cat. No. 74104) for clean up 
of RNA according to manufacturer's instructions. DNase treatment (10 U/reaction) was performed according to the Qiagen RNeasy kit (Cat. No. 74104). Concentrations of high purity RNA were measured using Nanodrop (Thermo Fisher Scientific, ND-2000). Reverse transcription to generate cDNA was performed using qscript mastermix (Quanta- Bio Cat. No. 101414-106) as recommended by the manufacturer using 750 ng of RNA template. Resulting cDNA was diluted 10x and used in the real-time PCR reaction using an Applied Biosystems Step One Plus thermocycler. Relative mRNA levels were calculated based on the delta-delta-CT method using the Applied Biosystems Step One Plus PCR System as we have previously described ${ }^{72-77}$. Primers used for qPCR are available upon request.

\section{Oral Glucose Tolerance Testing (OGTT)}

The mice were fasted for $4 \mathrm{~h}$ before the tests. OGTT was performed after a single gavage of glucose, $2.5 \mathrm{~g}$ per kg body weight for standard diet and $1 \mathrm{~g}$ per kg body weight for HFD and ob/ob studies. Blood glucose was then measured before (0 min) and after the injection (15, 30, 60, 120 min) using a OneTouch® SelectSimple ${ }^{\text {TM }}$ glucometer (LifeScan Inc., China).

\section{Measurement of Plasma Hormone and Lipid Levels}

Plasma insulin (EZRMI-13K, EMD Millipore) and corticosterone (501320, Cayman Chemical) levels were measured by ELISA. Plasma non-esterified fatty acid (HR Series NEFA-HR, Wako) and triglyceride (L-Type Triglyceride M, Wako) levels were measured using enzymatic assays according to manufacturer's instructions.

\section{Indirect Calorimetry}

To measure the effects of IMC on energy expenditure and physical activity mice were housed in metabolic cages (Oxymax CLAMS, Columbus Instruments) for indirect calorimetry 
measurements at room temperature $\left(22^{\circ} \mathrm{C}\right)$. Mice were acclimated to the home cage system for 72 hours prior to data collection, and data were analyzed as previously described ${ }^{72-74}$.

\section{Cecal Microbiome Analyses}

Snap frozen cecal DNA was isolated using the MO BIO Powersoilß-htp 96 well soil DNA isolation kit according to manufacturer's instructions. Region-specific primers $(515 \mathrm{~F} / 806 \mathrm{R})$ were used for amplifying the V4 region of the bacterial 16S rRNA gene for high throughput sequencing using the Illumina HiSeq platform, paired end 150bp run. The reverse amplicon primer contains a 12base Golay barcode sequence unique to each well that allows sample pooling for sequencing ${ }^{78-}$ 81. More information can be found at the Earth Microbiome Project 16S rRNA Amplification Protocol where our protocols were adapted from: http://www.earthmicrobiome.org/empstandard-protocols/16s/. Each sample was amplified in triplicate using 5 PRIME HotMaster Mix 2.5X (VWR 10847-708), combined, verified by $1.5 \%$ agarose gel and quantitated using Pico Green dsDNA Assay Kit (Thermofisher P7589). Samples were pooled (250 ng) and cleaned using the UltraClean PCR Clean-Up Kit protocol (Mo-Bio 12500-100). The quantified amplicons were sequenced with the Illumina HiSeq 2500 at the Broad Stem Cell Research Center at the University of California - Los Angeles on two lanes. The sequences were analyzed using the open source python software package Quantitative Insights Into Microbial Ecology (QIIME) version 1.9.1 $1^{78,83}$ using default parameters for each step, except where specified. Demultiplexed sequences were aligned and clustered into operational taxonomic units (OTUs) based on their sequence similarity ( $97 \%$ identity) using the SortMeRNA/SumaClust open reference based OTU picking protocol in QIIME. Representative sequences for each OTU were aligned using PyNAST (a python-based implementation of NAST $^{84}$ in Qiime and the Greengenes 11 database ${ }^{85}$. $38,826,537$ total reads were generated after removal of singleton reads and rare $(<0.01 \%$ of total reads) OTUs, with an average of 776,531 reads per sample. Samples were rarefied to the 
depth of the sample with the lowest number of reads $(86,941$ sequences/sample) for beta diversity assessment only. Beta diversity was assessed using weighted UniFrac in QIIME. Adonis statistical test with 1,000 permutations was used to determine the strength and statistical significance of sample groupings. LEfSe was used with default parameters on OTU tables to determine taxa that best characterize each population ${ }^{86}$. Significant differences in relative abundance of taxa between groups and correlations with physiological parameters were assessed using the ALDEx2 package implemented in $R^{87,898}$. Data were adjusted for false discovery rate using the Benjamini-Hochberg procedure and an adjusted $p$-value of $p<0.05$ was considered statistically significant. All other plots were carried out using R (r-project.org).

\section{Untargeted Metabolomics}

Moue plasma samples were prepared for untargeted metabolomics by diluting each plasma sample 1:20 in chilled methanol containing 5 internal standards as listed in the table below. The samples were then centrifuged at $14,000 \mathrm{~g}$ for 20 minutes to precipitate out the protein pellet. The supernatant was recovered and subjected to LC-MS analysis. One-microliter aliquots taken from each sample were pooled and this QC standard was analyzed every 10 injection. The untargeted metabolomics was performed by injecting $7 \mathrm{uL}$ of each sample onto a $10 \mathrm{~cm} \mathrm{C18}$ column (Thermo Fisher CA) coupled to a Vanquish UHPLC running at $0.25 \mathrm{~mL} / \mathrm{min}$ using water and $0.1 \%$ formic acid as solvent $A$ and acetonitrile and $0.1 \%$ formic acid as solvent $B$. The 15min gradient used is given below. The Orbitrap Q Exactive HF was operated in positive and negative electrospray ionization modes in different LC-MS runs over a mass range of 50-750 Da using full MS at 120,000 resolution. Data dependent acquisitions were obtained on the pooled QC sample. The DDA acquisition (DDA) include MS full scans at a resolution of 120,000 and HCD MS/MS scans taken on the top 10 most abundant ions at a resolution of 30,000 with dynamic exclusion of 4.0 seconds and the apex trigger set at 2.0 to 4.0 seconds. The resolution 
of the MS2 scans were taken at a stepped NCE energy of 20.0, 30.0 and 45.0. XCMS was used to deconvolute the data using 5 ppm consecutive scan error, 5 to 60 seconds as minimum and maximum peak width, S/N threshold of 10 , and span of 0.2 in positive mode and span of 0.4 in negative mode for retention time correction. The resulting peak table was further analyzed via MetaboLyzer. Briefly, the ion presence threshold was set at 0.7 in each study group. Data were then log-transformed and analyzed for statistical significance via non-parametric Mann-Whitney U-test (FDR corrected p-value <0.05). lons present in just a subset of samples were analyzed as categorical variables for presence status via Fisher's exact test. All p-values were corrected via the Benjamini-Hochberg step-up procedure for false discovery rate (FDR) correction. The data was then utilized for PCA, putative identification assignment, and pathway enrichment analysis via KEGG. In this dataset 7665 spectral features were detected, from which 1151 features were putatively assigned an identification in HMDB within a pre-defined 7ppm $\mathrm{m} / \mathrm{z}$ error window. Also, the MS/MS spectra of 120 of these features matched with a score of greater than $50 \%$ to 120 unique compounds on the mzCloud database. Given the complicated nature of comparing the global metabolome across two treatment groups and 6 circadian time points, we used an algorithm called Selective Paired Ion Contrast Analysis (SPICA) ${ }^{89}$, to reveal subtle differences the plasma metabolome kinetically. Pairwise analysis was conducted between all adjacent timepoints (ZT2, ZT6, ZT10, ZT14, ZT18, and ZT22) for each treatment group (chow control versus chow $+\mathrm{IMC}$ ), resulting in a total of 10 comparisons made (5 for chow and 5 for chow + IMC). Differences in the global plasma metabolome for each pairwise comparison were quantified via receiver operating characteristic (ROC) curve construction and area under the curve (AUC) calculations via Monte Carlo cross validation procedures in SPICA ${ }^{89}$. This analysis revealed that while all adjacent timepoints were roughly equally differentiated in the chow control data with an AUC averaging 0.884, this was not the case in the IMC-treated group. The AUC calculated for the IMC-treated group when comparing T10 vs. T14 was much greater than that 
of the chow-fed control group at the same time points (0.964 vs. 0.869$)$, meaning the differences in the plasma metabolome between these two timepoints were much more pronounced in the IMC-treated mice compared to chow controls. Furthermore, The AUCs calculated for the subsequent two timepoint comparisons (T14 vs. T18 and T18 vs. T22) were much lower in the IMC-treated group (0.624 and 0.615 , respectively) when compared to the chow control group (0.842 and 0.899, respectively), implying that the T14, T18, and T22 timepoints were poorly differentiated by IMC treatment. As a result, subsequent data analyses focused on this T10 to T14 transition, which also coincided with when the mice began to eat. The statistically significant positively-charged and negatively-charged spectral features which were present in more than $70 \%$ of the samples at ZT14 are shown in red in the volcano plots of Figure 4 . These features were then putatively identified in the Human Metabolome and the KEGG databases using their accurate mass-to-charge $(\mathrm{m} / \mathrm{z})$ values within a $7 \mathrm{ppm}$ error window. The KEGG annotated pathways associated with these putative metabolites were then identified. Figure 4c represents such KEGG pathways associated with the negatively charged spectral features in this study. This figure displays the KEGG metabolic pathways with the highest statistically significance to which the ions were assigned. The blue and red bars are the unadjusted and the FDR (false discovery rate)-adjusted -log of $p$-values respectively, while the orange line marks the significance threshold. This figure shows that lipid metabolism is the most perturbed metabolic pathway at ZT14.

\section{Data Analyses for Circadian Rhymicity (Cosinor Analyses)}

A single cosinor analysis was performed as previously described ${ }^{90-92}$. Briefly, a cosinor analysis was performed on each sample using the equation for cosinor fit as follows:

$Y(t)=M+A \cos (2 \pi / T+\phi)$ 
where $\mathrm{M}$ is the MESOR (Midline Statistic of Rhythm, a rhythm adjusted mean), $\mathrm{A}$ is the amplitude (a measure of half the extent of the variation within the cycle), $\phi$ is the acrophase (a measure of the time of overall highest value), and $\mathrm{T}$ is the period. The fit of the model was determined by the residuals of the fitted wave. After a single cosinor fit for all samples, linearized parameters were then averaged across all samples allowing for calculation of delineralized parameters for the population mean. A 24-h period was used for all analysis. Comparison of population MESOR, amplitude, and acrophase were performed as previously described ${ }^{28}$. Comparisons are based on F-ratios with degrees of freedom representing the number of populations and total number of subjects. All analysis was done in R v4.0.2 using the cosinor and cosinor2 packages ${ }^{93-95}$.

\section{Statistical Analysis}

All data were analyzed using either one-way or two-way analysis of variance (ANOVA) where appropriate, followed by either a Tukey's or Student's t tests for post hoc analysis. Differences were considered significant at $p<0.05$. All mouse data analyses were performed using Graphpad Prism 6 (La Jolla, CA) software.

\section{ACKNOWLEDGEMENTS}

This work was supported by National Institutes of Health grants R01 HL120679 (J.M.B.), P01 HL147823 (J.M.B., S.L.H.), P50 AA024333 (J.M.B), U01 AA026938 (J.M.B.), P50 CA150964 (J.M.B.), R01 HL103866 (S.L.H.), R01 HL147883 (A.J.L.), R01 HL144651 (A.J.L. and Z.W.), R01 HL130819 (Z.W.), F32 DK122623 (C.M.G.), T32 DK007307 (C.M.G.), a Leducq Transatlantic Networks of Excellence Award (S.L.H.), and the American Heart Association (Postdoctoral Fellowships 17POST3285000 to R.N.H and 15POST2535000 to R.C.S). Development of some of the mass spectrometry methods reported here were supported by generous pilot grants from the Clinical and Translational Science Collaborative of Cleveland 
(4UL1TR000439) from the National Center for Advancing Translational Sciences (NCATS) component of $\mathrm{NIH}$ and the NIH Roadmap for Medical Research, the Case Comprehensive Cancer Center (P30 CA043703), the VeloSano Foundation, and a Cleveland Clinic Research Center of Excellence Award.

\section{COMPETING FINANCIAL INTERESTS.}

R.C.S., C.M.G., R.N.H., A.L.B., A.B., C.F., K.K.F., F.M.A., D.F., A.D.G., C.N., A.M., J.T.A., M.M., M.G., B.W., T.D.M., A.R.A., G.S., A.K., A.J.L., and J.M.B. all declare no competing financial interests. Z.W. and S.L.H. report being named as co-inventor on pending and issued patents held by the Cleveland Clinic relating to cardiovascular diagnostics and therapeutics. S.L.H. reports being a paid consultant for Procter \& Gamble, having received research funds from Procter \& Gamble, and Roche Diagnostics, and being eligible to receive royalty payments for inventions or discoveries related to cardiovascular diagnostics or therapeutics from Cleveland Heart Lab and Procter \& Gamble. J.C.G-G is an employee of The Procter \& Gamble Co. J.A.B. reports being eligible to receive royalty payments for inventions or discoveries related to cardiovascular therapeutics from the Proctor \& Gamble Co.

\section{AUTHOR CONTRIBUTIONS}

R.C.S., C.M.G., and J.M.B. planned the project, designed experiments, analyzed data, and wrote the manuscript; A.K., J.C.G-G., A.J.L., and S.L.H. help design experiments and provided useful discussion directing collaborative aspects of the project; R.C.S., C.M.G., R.N.H., A.L.B., A.B., C.F., K.K.F., F.M.A., D.F., A.D.G., C.N., A.M., J.A.B., J.T.A., M.M., M.G., B.W., T.D.M., A.R.A., G.S., A.K., A.J.L., and J.M.B. either conducted mouse experiments, performed biochemical workup of mouse tissues, analyzed data, and aided in manuscript preparation; All authors were involved in the editing of the final manuscript. 


\section{LITERATURE CITED:}

1. Bäckhed, F. et al. Gut microbiota as an environmental factor that regulates fat storage. Proc. Natl. Acad. Sci. U S A. 101, 15718-15723 (2004).

2. Ley, R.E. et al. Obesity alters gut microbial ecology. Proc. Natl. Acad. Sci. USA. 102, 1107011075 (2005).

3. Turnbaugh, P.J. et al. An obesity-associated gut microbiome with increased capacity for energy harvest. Nature 444, 1027-1031 (2006).

4. Turnbaugh, P.J. et al. A core gut microbiome in obese and lean twins. Nature 457, 480-484 (2009).

5. Brown, J.M. \& Hazen, S.L. The gut microbial endocrine organ: bacterially derived signals driving cardiometabolic diseases. Annu Rev Med. 66, 343-359 (2015).

6. Sonnenburg J.L. \& Bäckhed F. Diet-microbiota interactions as moderators of human metabolism. Nature 535, 56-64 (2016).

7. Ajslev, TA., Anderson, C.S., Gamborg, M., Sørensen, T.I., \& Jess, T. Childhood overweight after establishment of the gut microbiota: the role of delivery mode, pre-pregnancy weight and early administration of antibiotics. Int. J. Obes. (Lond) 35, 522-529 (2011).

8. Cho, I. et al. Antibiotics in early life alter the murine colonic microbiome and adiposity. Nature 488, 621-626 (2012).

9. Canfora, E.E., Meex R.C.R., Venema K., \& Blaak E.E. Gut microbial metabolites in obesity, NAFLD and T2DM. Nat. Rev. Endocrinol. 15, 261-273 (2019).

10. Aron-Wisnewsky, J. et al. Gut microbiota and human NAFLD: disentangling microbial signatures from metabolic disorders. Nat. Rev. Gastroenterol. Hepatol. 17, 279-297 (2020).

11. Brown, J.M., \& Hazen, S.L. Microbial modulation of cardiovascular disease. Nat. Rev. Microbiol. 16, 171-181 (2018).

12. Knauf, F., Brewer, J.R., \& Flavell, R.A. Immunity, microbiota and kidney disease. Nat. Rev. Nephrol. 15, 283-274 (2019).

13. Marques F.Z., Mackay C.R., \& Kaye D.M. Beyond gut feelings: how the gut microbiota regulates blood pressure. Nat. Rev. Cardiol. 15, 20-32 (2018).

14. McQuade, J.L., Daniel, C.R., Helmink, B.A., \& Wargo, J.A. Modulating the microbiome to improve therapeutic response in cancer. Lancet Oncol. 20, e77-e91 (2019). 
15. Aron-Wisnewsky, J., Clément, K., \& Nieuwdorp, M. Fecal microbiota transplantation: a future therapeutic option for obesity/diabetes? Curr. Diab. Rep. 19, 51.

16. Asgharian, H., Homayouni-Rad, A., Mirghafourvand, M., \& Mohammad-AlizadehCharandabi, S. Effect of probiotic yoghurt on plasma glucose in overweight and obese pregnant women: a randomized controlled clinical trial. Eur. J. Nutr. 59, 205-215 (2020).

17. Reijinders, D., et al. Effects of gut microbiota manipulation by antibiotics on host metabolism in obese humans: A randomized double-blind placebo-controlled trial. Cell Metab. 24, 63-74 (2016).

18. Madjd, A., et al. Comparison of the effect of daily consumption of probiotic compared with low-fat conventional yogurt on weight loss in healthy obese women following an energyrestricted diet: a randomized controlled trial. Am. J. Clin. Nutr. 103, 323-329 (2016).

19. Koutnikova, H., et al. Impact of bacterial probiotics on obesity, diabetes and non-alcoholic fatty liver disease related variables: a systematic review and meta-analysis of randomised controlled trials. BMJ Open 9, e017995 (2019).

20. Roberts, A.B., et al. Development of a gut microbe-targeted nonlethal therapeutic to inhibit thrombosis potential. Nat. Med. 24, 1407-1417 (2018).

21. Wang, Z., et al. Non-lethal inhibition of gut microbial trimethylamine production for the treatment of atherosclerosis. Cell 163, 1585-1595 (2015).

22. Gupta, N., et al. Targeted inhibition of gut microbial trimethylamine N-oxide production reduces renal tubulointerstitial fibrosis and functional impairment in a murine model of chronic kidney disease. Arterioscler. Thromb. Vasc. Biol. 40, 1239-1255 (2020).

23. Organ, C.L., et al. Nonlethal inhibition of gut microbial trimethylamine $\mathrm{N}$-oxide production improves cardiac function and remodeling in a murine model of heart failure. J. Am. Heart Assoc. 9, e016223 (2020).

24. Orman, M., et al. Structure-guided identification of a small molecule that inhibits anaerobic choline metabolism by human gut bacteria. J. Am. Chem. Soc. 141, 33-37 (2019).

25. Wang, Z., et al. Gut flora metabolism of phosphatidylcholine promotes cardiovascular disease. Nature 472, 57-63 (2011).

26. Koeth, R., et al. Intestinal microbiota metabolism of L-carnitine, a nutrient in red meat, promotes atherosclerosis. Nat. Med. 19, 576-585 (2013).

27. Zhu, W., et al. Gut microbial metabolite TMAO enhances platelet hyperreactivity and thrombosis risk. Cell 165, 111-124 (2016).

28. Zhu, W., Wang, Z., Tang, W.H.W., \& Hazen, S.L. Gut microbe-generated trimethylamine Noxide from dietary choline is prothrombotic in subjects. Circulation 135, 1671-1673 (2017). 
29. Bell, J.D., et al. Nuclear magnetic resonance studies of blood plasma and urine from subjects with chronic renal failure: identification of trimethylamine-N-oxide. Biochim. Biophys. Acta 1096, 101-107 (1991).

30. Tang, W.H., et al. Gut microbiota-dependent trimethylamine N-oxide (TMAO) pathway contributes to both development of renal insufficiency and mortality risk in chronic kidney disease. Circ. Res. 116, 448-455 (2015).

31. Tang, W.H., et al. Prognostic value of elevated levels of intestinal microbe-generated metabolite trimethylamine-N-oxide in patients with heart failure: refining the gut hypothesis. J. Am. Coll. Cardiol. 64, 1908-1914 (2014).

32. Trøseid, M., et al. Microbiota-dependent metabolite trimethylamine-N-oxide is associated with disease severity and survival of patients with chronic heart failure. J. Intern. Med. 277, 717720 (2015).

33. Bae, S., et al. Plasma choline metabolites and colorectal cancer risk in the Women's Health Initiative observational study. Cancer Res. 74, 7442-7452 (2014).

34. Xu, R., Wang, Q., \& Li, L. A genome-wide systems analysis reveals strong link between colorectal cancer and trimethylamine $\mathrm{N}$-oxide (TMAO), a gut microbial metabolite of dietary meat and fat. BMC Genomics Suppl. 7, S4 (2015).

35. Shan, Z., et al. Association between microbiota-dependent metabolite trimethylamine-Noxide and type 2 diabetes. Am. J. Clin. Nutr. 106, 888-894 (2017).

36. Miao, J., et al. Flavin-containing monooxygenase 3 as a potential player in diabetesassociated atherosclerosis. Nat. Commun. 6, 6498 (2015).

37. Craciun, S., \& Balskus, E.P. Microbial conversion of choline to trimethylamine requires a glycyl radical enzyme. Proc. Natl. Acad. Sci. USA 109, 21307-21312 (2012).

38. Everard, A., et al. Cross-talk between Akkermansia mucinophila and intestinal epithelium controls diet-induced obesity. Proc. Natl. Acad. Sci. USA 110, 9066-9071 (2013).

39. Shin, N.R., et al. An increase in Akkermansia spp. population induced by metformin treatment improves glucose homeostasis in diet-induced obese mice. Gut 63, 727-735 (2014).

40. Org, E. et al. Genetic and environmental control of host-gut microbiota interactions. Genome Res. 25, 1558-1569 (2015).

41. Seldin, M.M., et al. Trimethylamine N-oxide promotes vascular inflammation through signaling of mitogen-activated protein kinase and nuclear factor kB. J. Am. Heart Assoc. 5, e002767 (2016).

40. Thaiss, C.A., et al. Transkingdom control of microbiota diurnal oscillations promote metabolic homeostasis. Cell 159, 514-529 (2014). 
41. Liang, X., Bushman, F.D., \& FitzGerald, G.A. Rhythmicity of the intestinal microbiota is regulated by gender and the host circadian clock. Proc Natl Acad Sci USA 112, 10479-10484 (2015).

42. Thaiss, C.A., et al. Microbiota diurnal rhythmicity programs host transcriptome oscillations. Cell 167, 1495-1510 (2016).

43. Wang, Y., et al. The intestinal microbiota regulate body composition through NFIL3 and the circadian clock. Science 357, 912-917 (2017).

44. Li, Q., et al. Synchronous evolution of an odor biosynthesis pathway and behavioral response. Curr. Biol. 23, 11-20 (2013).

45. Wallrabenstein, I., et al. Human trace amine-associated receptor TAAR5 can be activated by trimethylamine. PLoS One 8, e54950 (2013).

46. Zhu, Y., et al. Carnitine metabolism to trimethylamine by an unusual Rieske-type oxygenase from human microbiota. Proc Natl Acad Sci USA 111, 4268-4273 (2014).

47. Koeth, R.A., et al. gamma-Butyrobetaine is a proatherogenic intermediate in gut microbial metabolism of L-carnitine to TMAO. Cell Metab. 20, 799-812 (2014).

48. Li, X.S., et al. Untargeted metabolomics identifies trimethyllysine, a TMAO-producing nutrient precursor, as a predictor of incident cardiovascular disease risk. JCl Insight 3, e99096 (2018).

49. Lieber AD, et al. Loss of HDAC6 alters gut microbiota and worsens obesity. FASEB J. 33, 1098-1109 (2019).

50. Liu, S., \& Qin, P. Wang J. High-fat diet alters the intestinal microbiota in streptozotocininduced type 2 diabetic mice. Microorganisms 7, E176 (2019).

51. Moreira Júnior RE, et al. Interaction between high-fat diet and ethanol intake leads to changes on the fecal microbiome. J Nutr Biochem. 72, 108215 (2019).

52. Mak T.D., Laiakis E.C., Goudarz M., \& Fornace A.J. Selective paired ion contrast analysis: A novel algorithm for analyzing postprocessed LC-MS metabolomics data possessing high experimental noise. Anal. Chem. 87, 3177-3186 (2015).

53. Dehghan, P., Farhangi, M.A., Nikniaz, L., Nikniaz, Z., \& Asghari-Jafarabadi, M. Gut microbiota-derived metabolite trimethylamine $\mathrm{N}$-oxide (TMAO) potentially increases the risk of obesity in adults: An exploratory systematic review and dose-response meta-analysis. Obes. Rev. 21, e12993 (2020).

54. Maier, L., et al. Extensive impact of non-antibiotic drugs on human gut bacteria. Nature 555, 623-628 (2018).

55. Wu, H., et al. Metformin alters the gut microbiome of individuals with treatment-naïve type 2 diabetes, contributing to the therapeutic effects of the drug. Nat. Med. 23, 850-858 (2017). 
56. Chen, S., et al. Trimethylamine $\mathrm{N}$-oxide binds and activates PERK to promote metabolic dysfunction. Cell Metab. 30, 1141-1151 (2019).

57. Ly, S., Lee, D.A., Strus, E., Prober, D.A., \& Naidoo, N. Evolutionarily conserved regulation of sleep by the protein translational regulator PERK. Curr. Biol. 30, 1639-1648 (2020).

58. Bu, Y., et al. A PERK-miR-211 axis suppresses circadian regulators and protein synthesis to promote cancer cell survival. Nat. Cell Biol. 20, 104-115 (2018).

59. Bell, E., Prabakaran, S., Krishnan, P., Evans-Molina, C., \& Grant, M.B. Loss of diurnal oscillatory rhythms in gut microbiota correlates with changes in circulating metabolites in type 2 diabetic db/db mice. Nutrients 11, 2310 (2019).

60. Giskeødegård, G.F., Davies, S.K., Revell, V.L., Keun, H, \& Skene, D.J. Diurnal rhythms in the human urine metabolome during sleep and total sleep deprivation. Sci. Rep. 5, 14843 (2015).

61. Bollard, M.E., et al. Investigations into biochemical changes due to diurnal variation and estrus cycle in female rats using high-resolution (1)H NMR spectroscopy of urine and pattern recognition. Anal. Biochem. 295, 194-202 (2001).

62. Dixit, A., \& Roche, T.E. Spectrophotometric assay of the flavin-containing monooxygenase and changes in its activity in female mouse liver with nutritional and diurnal conditions. Arch. Biochem. Biophys. 233, 50-63 (1984).

63. Chen, M., et al. The molecular mechanism regulating diurnal rhythm of flavin-containing monooxygenase 5 in mouse liver. Drug Metab. Dispos. 47, 1333-1342 (2019).

64. Astafev, A.A., Patel, S.A., \& Kondratov, R.V. Caloric restriction effects on circadian rhythms in gene expression are sex dependent. Sci. Rep. 7, 9716 (2017).

65. Hughes, M.E., Hogenesch, J.B., \& Kornacker, K. JTK_Cycle: an efficient non-parametric algorithm for detecting rhythmic components in genome-scale datasets. J. Biol. Rhythms 25, 372-380 (2010).

66. Mistry, J.S., Abraham, D.J., Kozikowski, A.P., \& Hanin, I. Neurochemistry of Aging. 2. Design, Synthesis, and Biological Evaluation of Halomethyl Analogues of Choline with High Affinity Choline Transport Inhibitory Activity"; J. Med. Chem. 34, 2031-2036 (1991).

67. Wang, Z., et al. Measurement of trimethylamine-N-oxide by stable isotope dilution liquid chromatography tandem mass spectrometry. Anal. Biochem. 455, 35-30 (2014).

68. Dobin, A., et al. STAR: ultrafast universal RNA-seq aligner. Bioinformatics 29, 15-21 (2013).

69. Robinson, M.D., McCarthy, D.J., \& Smyth, G.K. edgeR: a bioconductor package for differential expression analysis of digital gene expression data. Bioinformatics 26, 139-140 (2010). 
70. Kolde, R. pheatma: Pretty Heatmaps. R Package Version. http://CRAN.Rproject.org/package=pheatmap (2015).

71. Yu, G., \& He, Q.Y. ReactomePA: A R/Bioconductor package for reactome pathway analysis and visualization. Mol. BioSystems 12, 477-479 (2016).

72. Lord, C.C., et al. Regulation of hepatic triacylglycerol metabolism by CGI-58 does not require ATGL co-activation. Cell Rep. 16, 939-949 (2016).

73. Thomas, G., et al. The serine hydrolase ABHD6 is a critical regulator of the metabolic syndrome. Cell Rep. 5, 508-520 (2013).

74. Schugar, R.C., et al. The TMAO-producing enzyme flavin-containing monooxygenase 3 regulates obesity and the beiging of white adipose tissue. Cell Rep. 19, 2451-2461 (2017).

75. Brown, J.M., et al. CGl-58 knockdown in mice causes hepatic steatosis but prevents dietinduced obesity and glucose intolerance. J. Lipid Res. 51, 3306-3315 (2010).

76. Brown, J.M., et al. Inhbition of Stearoyl-coenzyme A desaturase 1 dissociates insulin resistance and obesity from atherosclerosis. Circulation 118, 1467-1475 (2008).

77. Gromovsky, A.D., et al. Delta-5 fattty acid desaturase FADS1 impacts metabolic disease by balancing proinflammatory and proresolving lipid mediators. Arteriosclero. Thromb. Vasc. Biol. 38, 218-231 (2018).

78. Caporaso, J.G., et al. QIIME allows analysis of high-throughput community sequencing data. Nat. Methods 7, 335-336 (2010).

79. Caporaso, J.G., et al. Ultra-high-throughput microbial community analysis on the Illumina HiSeq and MiSeq platforms. ISME J 6, 1621-1624 (2012).

80. Costello, E.K., et al. Bacterial community variation in human body habitats across space and time. Science 326, 1694-1697 (2009).

81. Fierer, N., Hamady, M., Lauber, C.L., \& Knight, R. The influence of sex, handedness, and washing on the diversity of hand surface bacteria. Proc. Natl. Acad. Sci. USA 105, 17994-17999 (2008).

82. Hamady, M., Walker, J.J., Harris, J.K., Gold, N.J., \& Knight, R. Error-correcting barcoded primers for pyrosequencing hundreds of samples in multiplex. Nat. Methods 5, 235-237 (2008).

83. Kuczynski J, et al. Using QIIME to analyze 16S rRNA gene sequences from microbial communities. Curr. Protoc. Bioinformatics Chapter 10: Unit 1017 (2011). 
84. Caporaso, J. G., et al. PyNAST: a flexible tool for aligning sequences to a template alignment. Bioinformatics 26, 266-267 (2010).

85. DeSantis, T. Z., et al. Greengenes, a Chimera-Checked 16S rRNA Gene Database and Workbench Compatible with ARB. Appl. Environ. Microbiol. 72, 5069-5072 (2006).

86. Segata, N., et al. Metagenomic biomarker discovery and explanation. Genome Biol. 12, R60 (2011).

87. Fernandes, A. D., et al. ANOVA-like differential expression (ALDEx) analysis for mixed population RNA-SEq. PLoS One, 8, e67019 (2013).

88. Fernandes, A. D., et al. (2014). Unify- ing the analysis of high-throughput sequencing datasets: Characterizing RNA-seq, $16 \mathrm{~S}$ rRNA gene sequencing and selective growth experiments by compositional data analysis. Microbiome, 2, 15 (2014).

89. Mak T.D., Laiakis E.C., Goudarz M., \& Fornace A.J. Selective paired ion contrast analysis: A novel algorithm for analyzing postprocessed LC-MS metabolomics data possessing high experimental noise. Anal. Chem. 87, 3177-3186 (2015).

90. Cornelissen G. Cosinor-based rhythmometry. Theor. Biol. Med. Model. 11, 11-16, (2014).

91. Nelson, W., Tong, Y.L., Lee, J.K., \& Halberg, F. Methods for cosinorrhythmometry. Chronobiologia 6, 305-323, 197 (1979).

92. Bingham, C., Arbogast, B., Guillaume Cornélissen, G., Lee, J.K. \& Halberg, F. Inferential Statistical Methods for Estimating and Comparing Cosinor Parameters. Chronobiologia 9, 397439 (1982).

93. Sachs, M.C., et al. Estimating mean annual 25-hydroxyvitamin D concentrations from single measurements: the Multi-Ethnic Study of Atherosclerosis. Am. J. Clin. Nutr. 97, 1243-1251 (2013).

94. Sachs, M.C. cosinor: Tools for estimating and predicting the cosinor model. R package version 1.1. https://CRAN.R-project.org/package=cosinor (2014).

95. Mutak, A. cosinor2: Extended Tools for Cosinor Analysis of Rhythms. R package version 0.2.1. https://CRAN.R-project.org/package=cosinor2 (2018). 


\section{FIGURE LEGENDS}

Figure 1. Small Molecule Choline TMA Lyase Inhibition Improves Obesity. Panels a-f and g-I represent data from control and IMC-treated HFD-fed and ob/ob mice, respectively. a and $\mathbf{g}$, plasma TMA levels. $\mathbf{b}$ and $\mathbf{h}$, plasma TMAO levels. $\mathbf{c}$ and $\mathbf{i}$, biweekly body weights. $\mathbf{d}$ and $\mathbf{j}$, biweekly food consumption. $\mathbf{e}$ and $\mathbf{k}$, glucose tolerance test. f, plasma insulin levels. I, average oxygen consumption. In panels a-e and $\mathbf{g - k}$, groups were compared using t-tests. In panels $\mathbf{f}$ and I, groups were compared using two-way ANOVA with Tukey's multiple comparisons test. Significance is defined as: ${ }^{*}, \mathrm{P}<0.05 .{ }^{* *}, \mathrm{P}<0.01 .{ }^{* * *}, \mathrm{P}<0.001,{ }^{* * *}, \mathrm{P}<0.0001 . \mathrm{n}=6-10$ per group.

Figure 2. Small Molecule TMA Lyase Inhibition Promotes Beneficial Remodeling of the Gut Microbiome. Panels a-e and f-i represent data from control and IMC-treated HFD-fed and ob/ob mice, respectively. a and $\mathbf{f}$, Principal co-ordinate analysis plot of microbiota profiles built from weighted Unifrac distances. Each point represents a single sample from a single mouse. Positions of points in space display dissimilarities in the microbiota, with points further from one another being more dissimilar. $\mathbf{b}$ and $\mathbf{g}$, Barplot of cecal microbiota at the phylum level. Each bar represents an individual mouse and each color an individual phyla. c. Relative abundance of Firmicutes, Bacteroidetes and the Firmicutes to Baceteroidetes ratio. The boxes represent the $25^{\text {th }}$ and $75^{\text {th }}$ quartiles, and the line displays the median value within each group. Points extending beyond the lines are outliers defined as values greater or less than 1.5 times the interquartile range. $\mathbf{d}$ and $\mathbf{h}$, Linear discriminatory analyses plot of taxa differing significantly with IMC treatment. e and i, Correlation between taxa and plasma TMA levels, body weight, and glucose tolerance (HFD-fed mice only). Values in both $\mathrm{X}$ and $\mathrm{Y}$ directions are plotted as mean \pm SEM. 
Figure 3. Nodes Within the Metaorganismal TMAO Pathway Show Diurnal Rhythmicity and Choline TMA Lyase Inhibition Alters the Host Circadian Clock. Wild type male C57BL/6J mice were necropsied at 4 hour intervals to collect plasma and tissue over a 24 hour period. a. Plasma choline, L-carnitine, trimethylamine (TMA); trimethylamine N-oxide (TMAO) were quantified by LC-MS/MS; hepatic expression of Fmo3 mRNA or gastrocnemius muscle expression of Taar5 mRNA was quantified via qPCR $(n=7-8)$. b. Wild type male C57BL/6J mice were fed chow or chow supplemented with the choline TMA lyase inhibitor iodomethylcholine (IMC) for 7 days. Mice were then necropsied at 4 hour intervals to collect liver, gonadal white adipose tissue, or gastrocnemius skeletal muscle. We then performed qPCR to examine the mRNA expression in liver, white adipose, and gastrocnemius skeletal muscle of key circadian clock regulators including aryl hydrocarbon receptor nuclear translocator like (Arntl; BMAL1), nuclear receptor subfamily 1 group D member 1 (Nr1d1; RevErb $\alpha)$, cryptochrome 1 (Cry1), or period $2(P e r 2)(\mathrm{n}=7-8)$. Significance $(p<0.05)$ between diet groups at specified Zeitgeber $(Z T)$ time points were compared using Student's t-tests.

Figure 4. Small Molecule Inhibition of Choline TMA Lyases Impacts Circadian Oscillations in Choline Containing Phospholipids in the Host Circulation. Wild type male C57BL/6J mice were fed chow or chow supplemented with the choline TMA lyase inhibitor iodomethylcholine (IMC) for 7 days. Mice were then necropsied at 4 hour intervals to collect blood and tissue, and plasma time points were subjected to LC-MS/MS-based untargeted metabolomics $(n=7-8)$. a. Selective Paired Ion Contrast Analysis (SPICA) ${ }^{51}$ was used to identify time points where the plasma metabolome was most dramatically altered by IMC treatment compared to zeitgebermatched controls. Pairwise analysis was conducted between all adjacent timepoints for the control and IMC treatment groups, resulting in a total of 10 comparisons made ( 5 for Chow and 
5 for Chow $+I M C)$. Differences in the global plasma metabolome for each pairwise comparison were quantified via receiver operating characteristic $(R O C)$ curve construction and area under the curve (AUC) calculations via Monte Carlo cross validation procedures in SPICA ${ }^{51}$ revealed that IMC most dramatically altered the plasma metabolome during the transition from ZT10 to ZT14. As a result, subsequent data analyses focused on this T10 to T14 transition, which also coincided with the light to dark phase transition and when the mice began to eat. b. Volcano plots of the significantly altered metabolites (red) in positive and negative ion mode at the ZT14 time point. c. Pathway analysis of data collected in negative ion mode at ZT14 reveals that IMC alters host lipid and lipoprotein metabolism among other metabolic pathways. d. Relative plasma levels of phosphatidylcholine (PC) and lysophosphatidylcholine (LPC) species are altered by IMC.

Figure 1 - figure supplement 1. Choline TMA Lyase Inhibition Increases Energy Expenditure and Alters Gene Expression in White Adipose Tissue. Related to main text Figure 1. Panels a-c represent indirect calorimetry data from control and IMC-treated $o b / o b$ mice. a. oxygen consumption. b. $\mathrm{CO}_{2}$ production. c. Respiratory exchange ratio collected in the Oxymax CLAMS metabolic cage system. d-f. C57BL6 mice fed a high fat diet (HFD) for 6 weeks to establish obesity (body weight $>35$ grams); after 6 weeks of DIO mice were continued on HFD alone of HFD containing IMC for another 10 weeks to test whether IMC can improve obesity-related phenotypes. $\mathbf{d}$. body weight curve. e. food consumption after drug administration. f. glucose tolerance testing 8 weeks following IMC administration. g-k. Male C57BL6/J mice were fed a HFD for 20 weeks and then gonadal white adipose tissue was collected for examination of global transcriptome alterations via RNA sequencing. g. Principal component analysis. h. heatmap of most significantly altered mRNA transcripts. i. Hallmark pathway 
analysis of differentially expressed genes (DEGs). j. volcano plot showing most significantly up and down regulated transcripts.

Figure 2 - figure supplement 1. TMA Lyase Inhibition Beneficially Improves Circadian Oscillations in Gut Microbial Communities. Related to main text Figure 2. Wild type male C57BL/6J mice were fed chow or chow supplemented with the choline TMA lyase inhibitor iodomethylcholine (IMC) for 7 days. Mice were then necropsied at 4 hour intervals to collect cecum for 16S-based microbiome analyses. a. Principal coordinate analysis (Bray-Curtis) comparing chow versus chow + IMC, light cycle versus dark cycle, and across all ZT time points. b. Identification of cyclic taxa whose relative abundance also differed significantly between groups (highlighted in yellow). FDR corrected P values for treatment effect (two-way ANOVA) are shown. Circadian rhythmicity of cecal microbial taxa was analyzed using JTK_Cycle. c. Relative abundance of key IMC-altered taxa that are circadian in nature $(\mathrm{n}=7-8)$; Significance between groups at specified zeitgeber $(\mathrm{ZT})$ time points were compared using Student's t-test ( $p$ $<0.05)$

Figure 3 - figure supplement 1. TMA Lyase Inhibition Alters the Circadian Rhythmicity of Host Metabolism-Associated Gene Expression. Related to main text Figure 3. Wild type male C57BL/6J mice were fed chow or chow supplemented with the choline TMA lyase inhibitor iodomethylcholine (IMC) for 7 days. Mice were then necropsied at 4 hour intervals to collect blood and metabolic tissues (liver and gastrocnemius skeletal muscle). a. Plasma levels of choline, trimethylamine (TMA), and trimethylamine $\mathrm{N}$-oxide (TMAO). b. The relative mRNA expression of forkhead box protein 01 (Foxo1), choline kinase $\alpha$ (Chk $\alpha)$, phosphatidylethanolamine N-methyltransferase (Pemt) in the liver over a 24-hour period. c. The relative mRNA expression of the TMA receptor trace amine-associated receptor 5 (Taar5), 
nuclear receptor subfamily 1 group D member 1 (Nr1d1; RevErb $\alpha$ ), and peroxisome proliferatoractivated receptor gamma coactivator $1 \alpha(\mathrm{Pgc} 1 \alpha)$ in the gastrocnemius muscle over a 24-hour period. d. Circadian parameters (MESOR, amplitude, and acrophase) were determined by cosinor analysis with a 24 hour period in plasma, liver, and skeletal muscle. The overall fit for each model is shown in Cosinor Detect, and a Wald multivariate test was used to compare circadian parameters between groups. All data represent mean -/+ S.E.M ( $\mathrm{n}=7-8$ per group).

Figure 4 - figure supplement 1. Choline TMA Lyase Inhibition Impact on the Circadian Rhythmicity of Host Hormone Levels and Gene Expression. Related to main text Figure 4. Wild type male C57BL/6J mice were fed chow or chow supplemented with the choline TMA lyase inhibitor iodomethylcholine (IMC) for 7 days. Mice were then necropsied at 4 hour intervals to collect blood/plasma. a. Plasma corticosterone levels were measured via ELISA. b. Plasma insulin levels were measured by ELISA. c. Plasma non-esterified fatty acid (NEFA) levels were measure via enzymatic assay. d. FoxO1 mRNA levels in epidydimal white adipose tissue (eWAT). e. Taar5 mRNA levels in gastrocnemius skeletal muscle. f. Taar5 mRNA levels in the heart. g. Taar5 mRNA levels in the olfactory bulb in chow-fed mice. h. Fmo3 mRNA level in epidydmal white adipose tissue (eWAT). Significance between groups $(n=7-8)$ at each zeitgeber time point were compared using Student's t-test $(p<0.05)$. 
bioRxiv preprint doi: https://doi org/10.1101/2020.12.04.411546; this version posted December 6. 2020. The copyright holder for this preprint (which was not certified by peer review) is the author/funder, who has granted bioRxiv a license to display the preprint in perpetuity. It is made available under aCC-BY 4.0 International license.

Figure 1
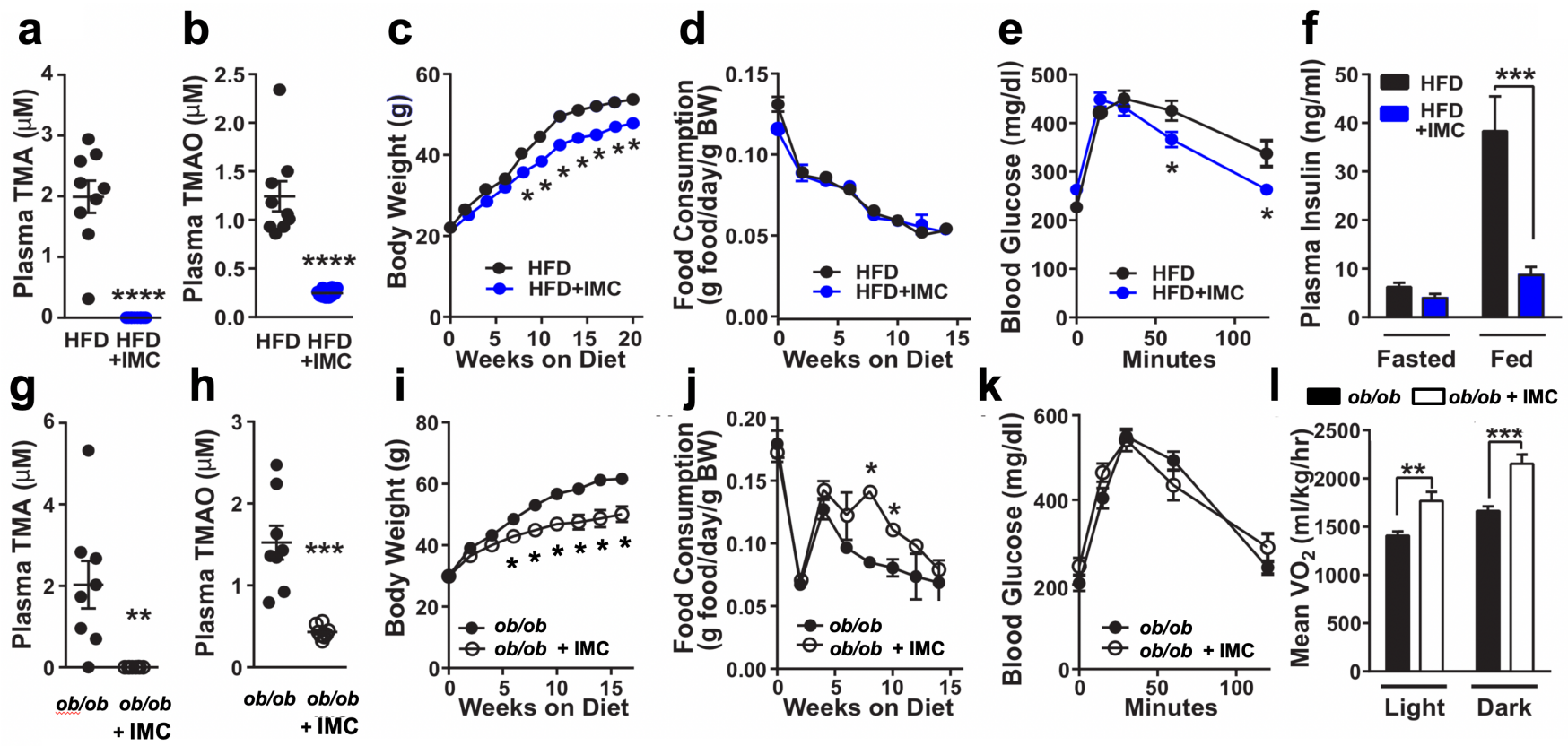
bioRxiv preprint doi: https://doi.org/10.1101/2020.12.04.411546; this version posted December 6, 2020. The copyright holder for this preprint (which was not certified by peer review) is the author/funder, who has granted bioRxiv a license to display the preprint in perpetuity. It is made available under aCC-BY 4.0 International license.

\section{Figure 2}

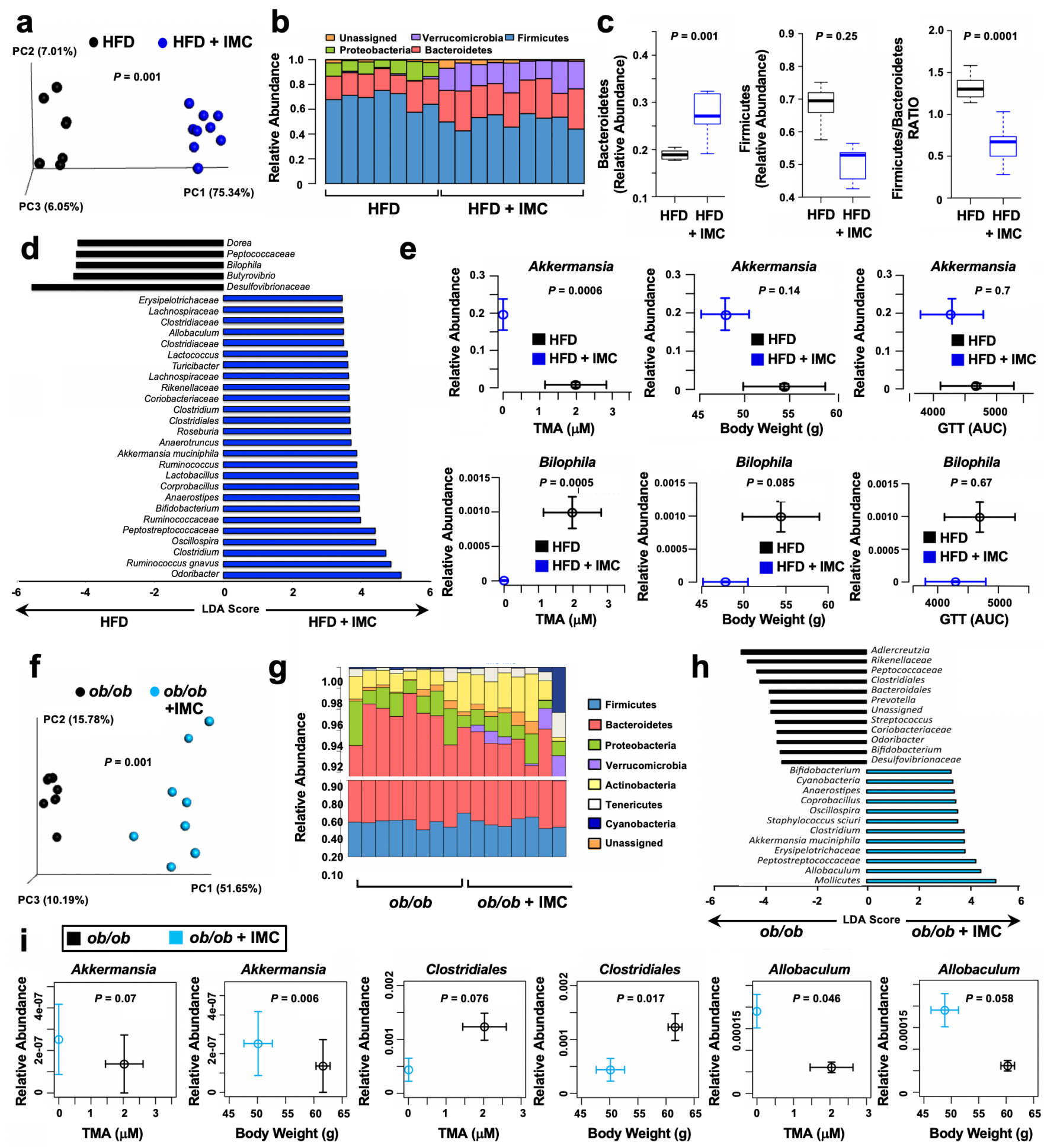


Figure 3
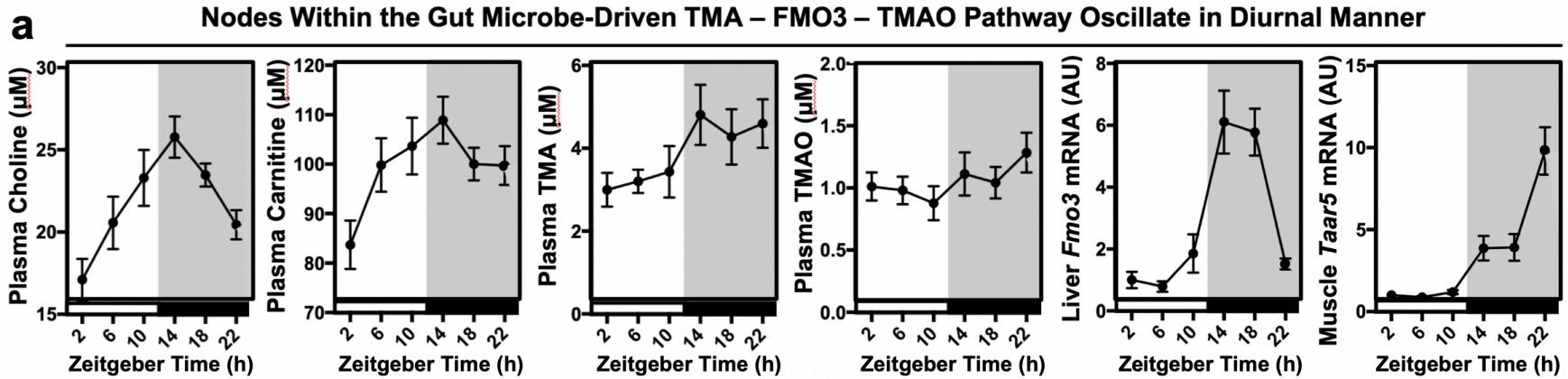

b

TMA Lyase Inhibition Reorganizes the Host Circadian Clock in a Tissue-Specific Manner

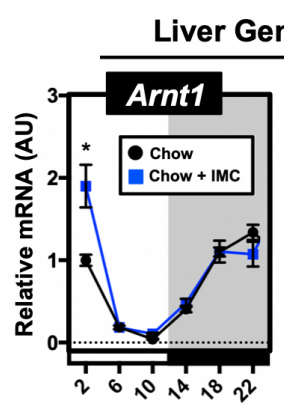

Zeitgeber Time (h)

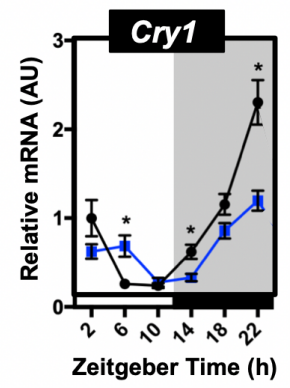

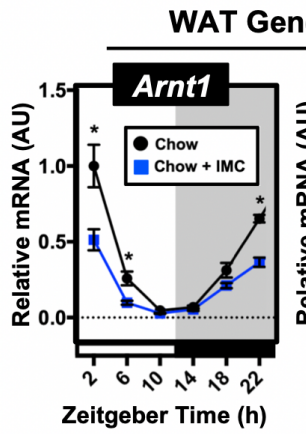

Cry1

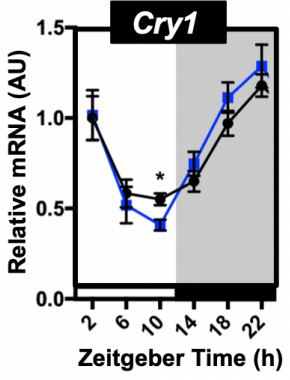

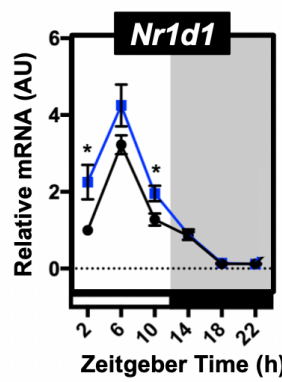

Per2

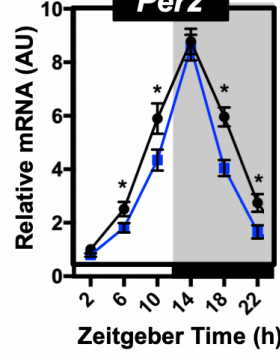

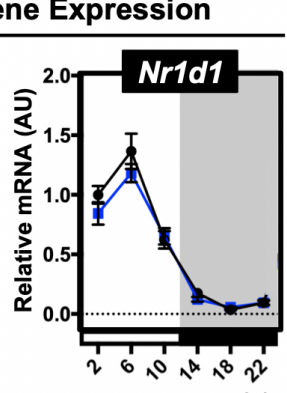

Zeitgeber Time (h)

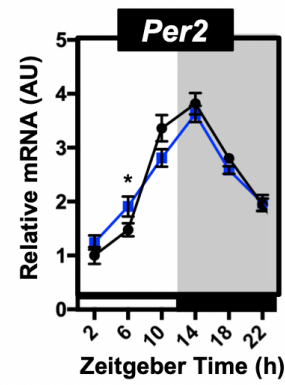

Skeletal Muscle Gene Expression

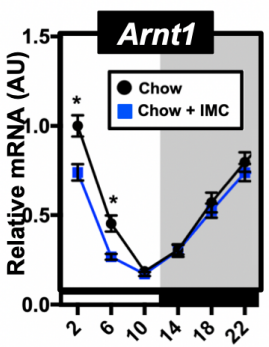

Zeitgeber Time (h)

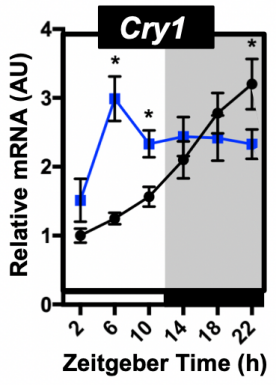

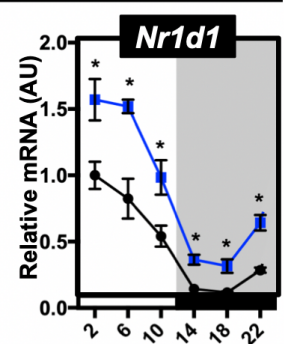

Zeitgeber Time (h)

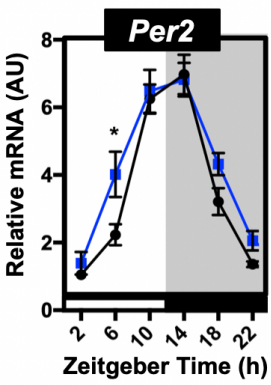


bioRxiv preprint doi: https://doi.org/10.1101/2020.12.04.411546; this version posted December 6, 2020. The copyright holder for this preprint (which was not certified by peer review) is the author/funder, who has granted bioRxiv a license to display the preprint in perpetuity. It is made available under aCC-BY 4.0 International license.

\section{Figure 4}

Predictive Model of IMC-Driven Alterations in Circulating Metabolites

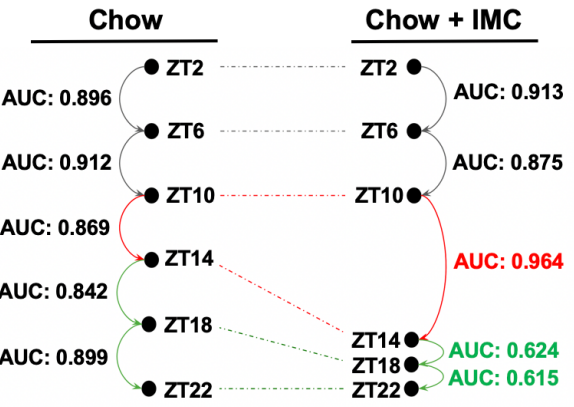

b Untargeted Metabolomic Signatures in Positive lon Mode (ZT14)

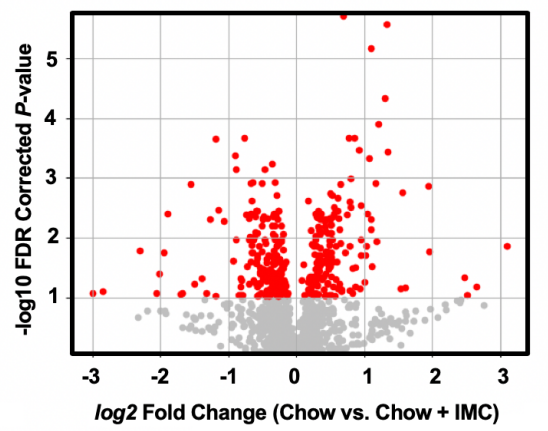

Untargeted Metabolomic Signatures in Negative lon Mode (ZT14)

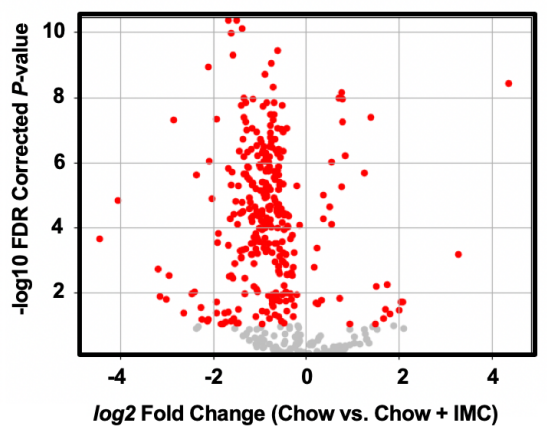

C Host Metabolic Pathways Altered by IMC -log10 $P$-value

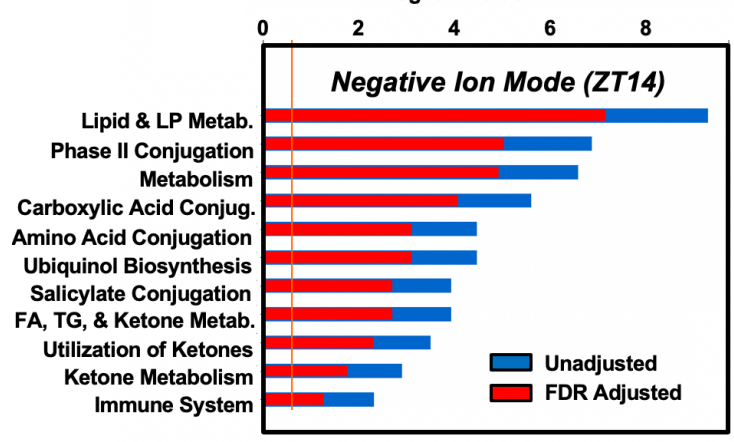

TMA Lyase Inhibition Alters the Diurnal Rhythms in Lysophosphatidylcholine (LPC) and Phosphatidylcholine (PC) Species

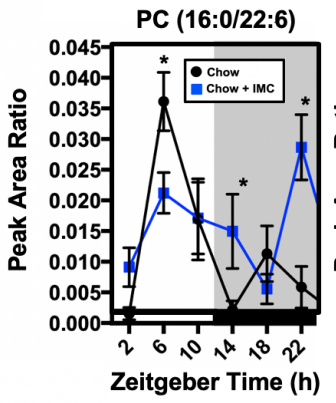

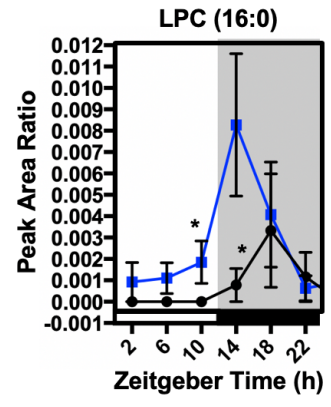


bioRxiv preprint doi: https://doi.org/10.1101/2020 12.04.411546; this version posted December 6, 2020. The copyright holder for this preprint (which was not certified by peer review) is the author/funder, who has granted bioRxiv a license to display the preprint in perpetuity. It is made available under aCC-BY 4.0 International license.

Figure 1 - figure supplement 1

a

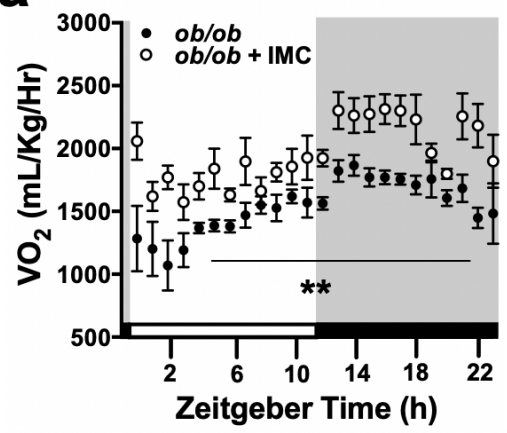

d

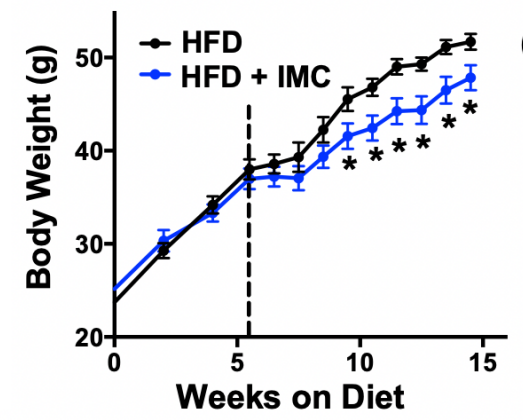

b
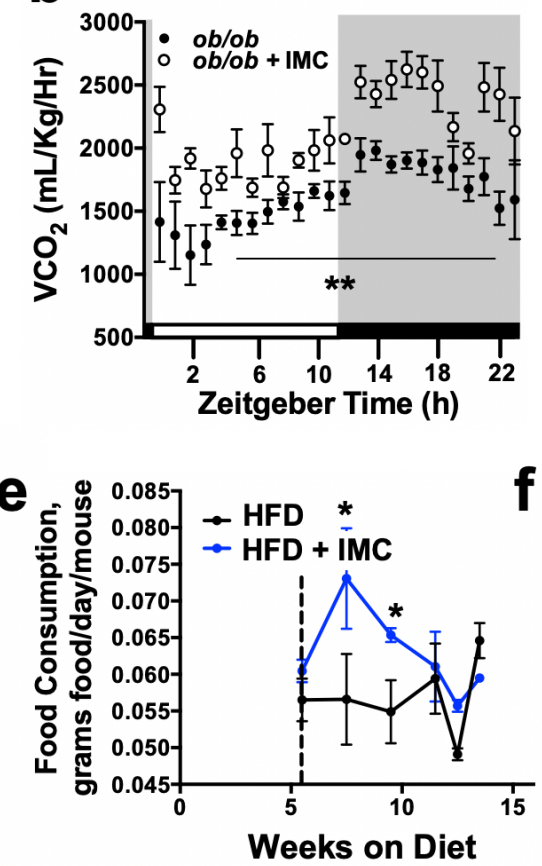

g

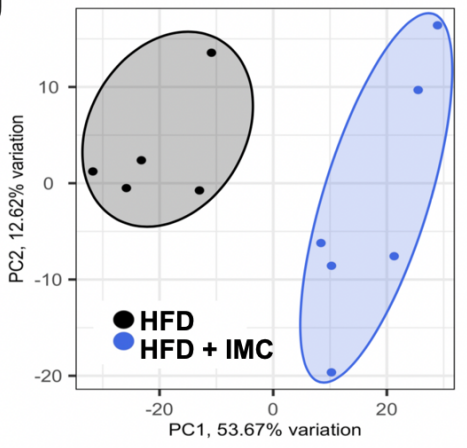

j

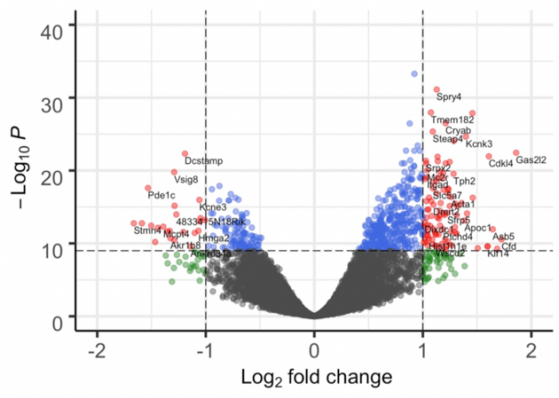

- NS $\bullet \log _{2} \mathrm{FC} \bullet p$-value $\bullet \mathrm{p}$-value and $\log _{2} \mathrm{FC}$ h

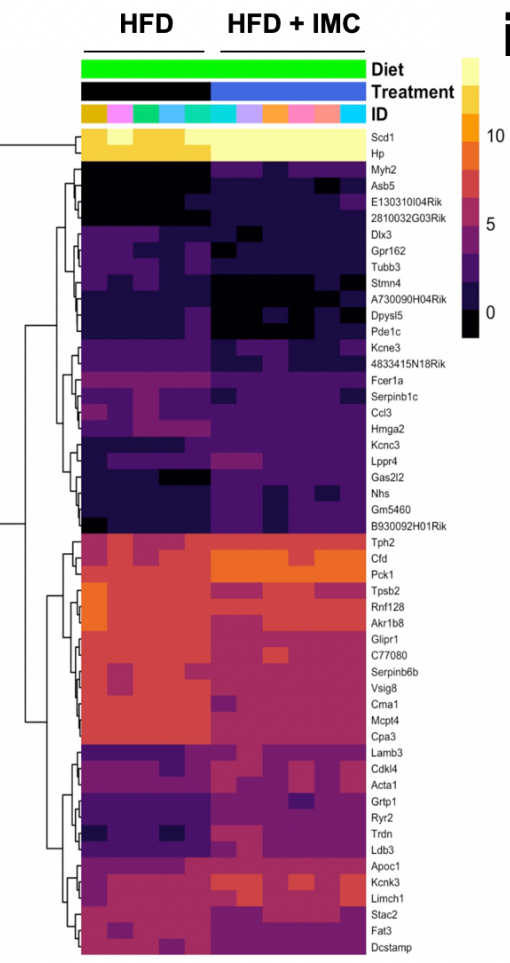

C
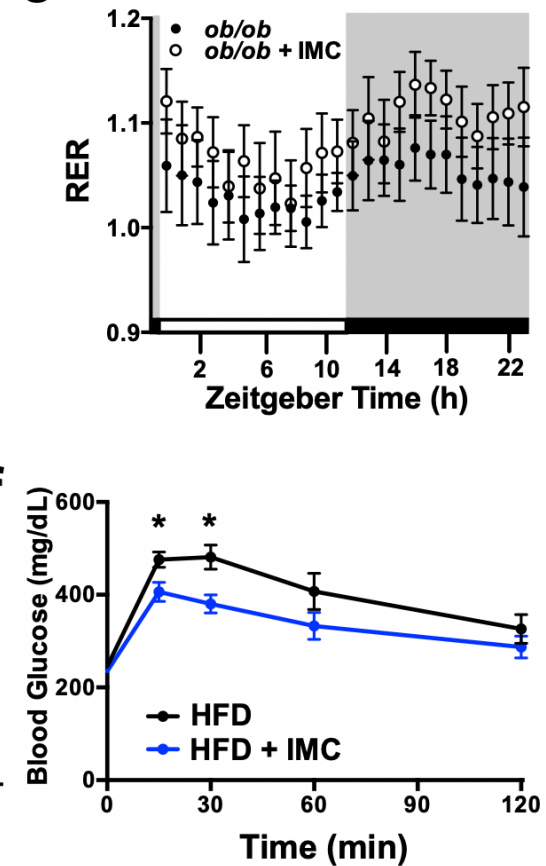

i
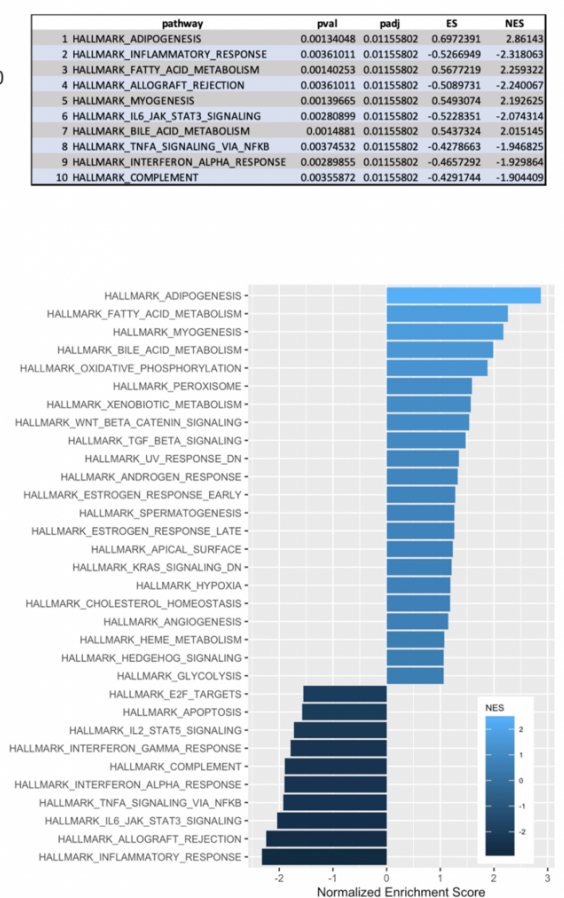
Figure 2 - figure supplement 1
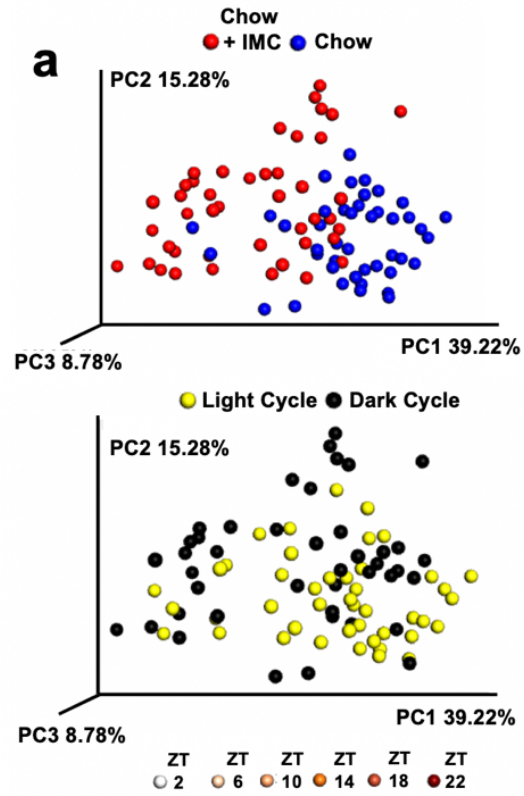

b Circadian Rhythmicity Analyzed by JTK_Cycle

\begin{tabular}{|lccc|}
\hline \multicolumn{1}{|c}{ Taxa } & P-value & Circadian in Chow & Circadian in IMC \\
\hline Coprococcus & $6.45 \mathrm{E}-15$ & No & No \\
Akkermansia mucinophila & $2.17 \mathrm{E}-14$ & No & No \\
\hline Clostridiales & $1.84 \mathrm{E}-11$ & No & Yes \\
Ruminococus gnavus & $8.62 \mathrm{E}-11$ & No & Yes \\
\hline Coprobacillus & $3.68 \mathrm{E}-09$ & No & No \\
Adlercreutzia & $4.14 \mathrm{E}-07$ & Yes & Yes \\
\hline Lachnospiraceae & $7.50 \mathrm{E}-05$ & Yes & No \\
Lachnospiraceae ruminococcus & $9.53 \mathrm{E}-05$ & Yes & No \\
\hline Bacteria & 0.00021281 & No & No \\
Clostridium & 0.00029686 & No & No \\
Dorea & 0.00029686 & No & No \\
Erysipelotrichaceae & 0.00033574 & No & No \\
Mogibacteriaceae & 0.00053423 & No & No \\
Turicibacter & 0.00183041 & No & No \\
Ruminococcaceae ruminococcus & 0.00249661 & No & No \\
Streptophyta & 0.00679781 & No & No \\
\hline Clostridiaceae & 0.0076506 & No & Yes \\
\hline Blautia producta & 0.02607991 & No & No \\
\hline Bacteriodales S24-7 & 0.03297143 & Yes & No \\
\hline Blautia & 0.03748415 & No & No \\
Unassigned & 0.04002213 & Yes & Yes \\
\hline
\end{tabular}

PC3 8.78\%

PC1 39.22\%

\section{Chow Chow + IMC}

C

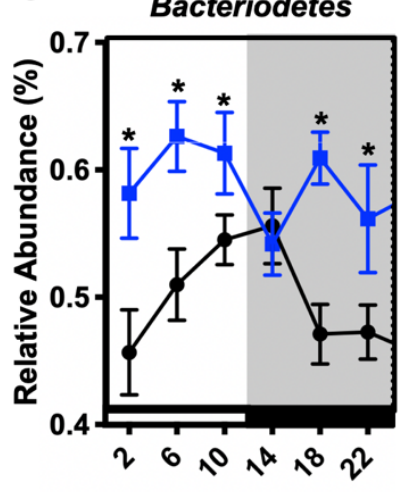

Zeitgeber Time (h)
Firmicutes

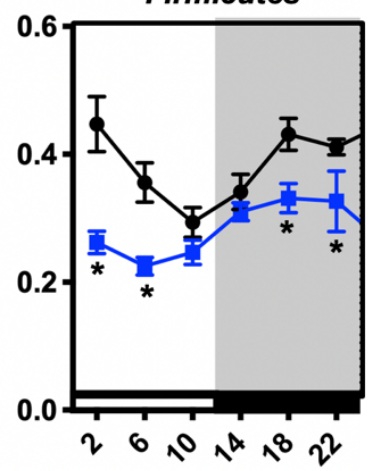

Zeitgeber Time (h)

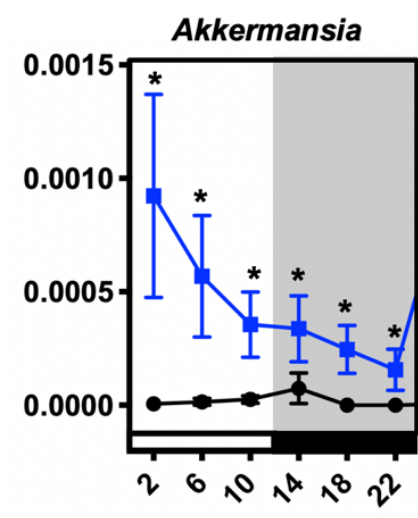

Zeitgeber Time (h)
Bacteroidetes, S24-7

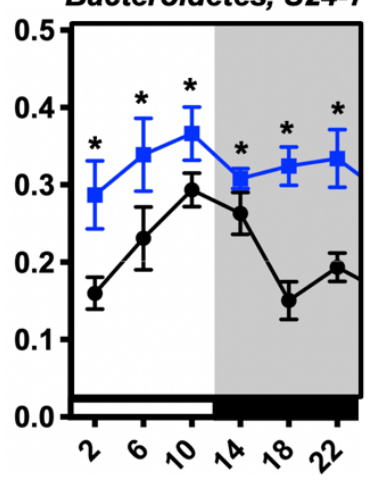

Zeitgeber Time (h) 
bioRxiv preprint doi: https://doi.org/10.1101/2020.12.04.411546; this version posted December 6, 2020. The copyright holder for this preprint (which was not certified by peer review) is the author/funder, who has granted bioRxiv a license to display the preprint in perpetuity. It is made available under aCC-BY 4.0 International license.

Figure 3 - figure supplement 1

a Effects of Gut Microbe-Targeted TMA Lyase Inhibition on Plasma Metabolites

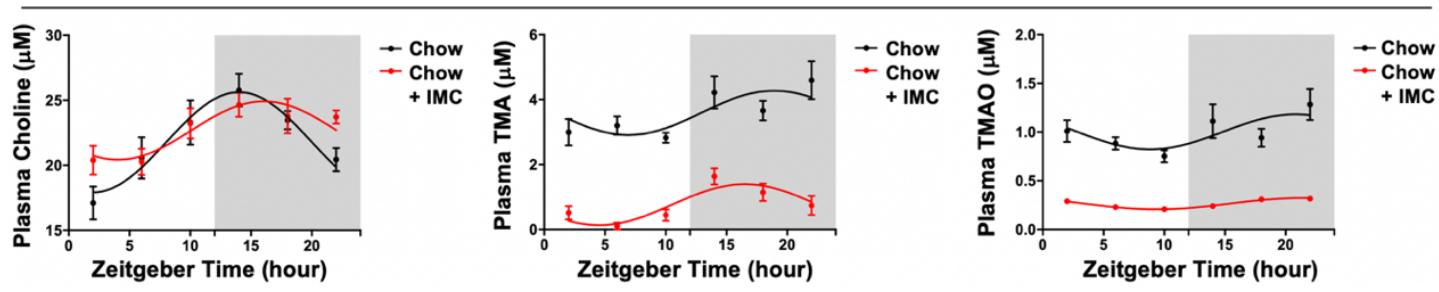

b Effects of Gut Microbe-Targeted TMA Lyase Inhibition on Metabolic Gene Expression in the Liver
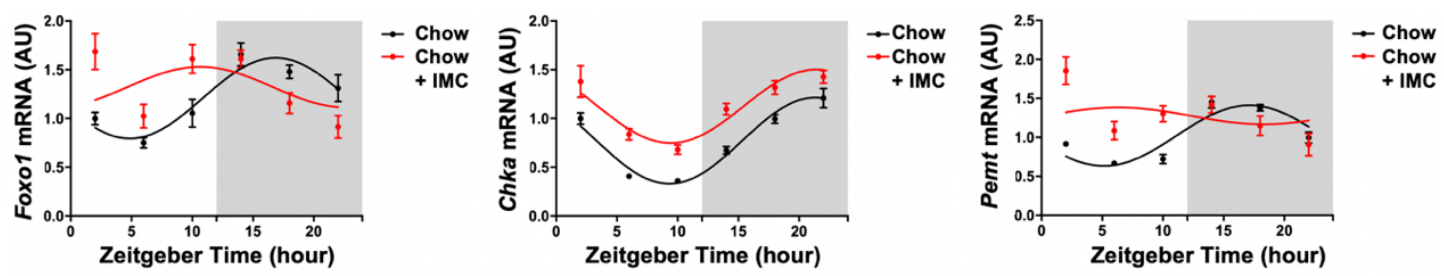

C Effects of Gut Microbe-Targeted TMA Lyase Inhibition on Gene Expression in Skeletal Muscle
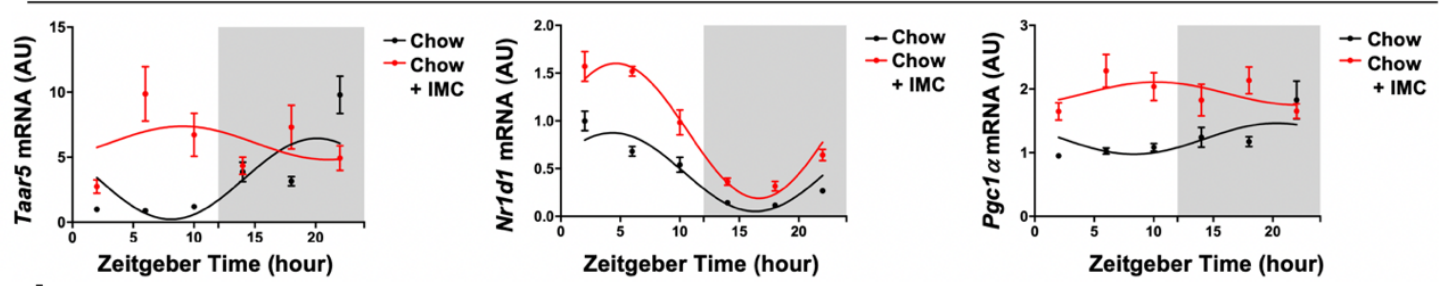

d

\begin{tabular}{|c|c|c|c|c|c|c|c|}
\hline & Group & Cosinor Detect & MESOR & Amplitude & Acrophase & MESOR-Test & Amplitude-Test \\
\hline \multirow{2}{*}{ Plasma Choline $(\mu \mathrm{M})$} & Chow & $7.87 \mathrm{E}-05$ & $21.78 \pm 1.85$ & $3.85 \pm 0.99$ & $14.02 \pm 1.53$ & \multirow{2}{*}{0.333} & \multirow{2}{*}{0.395} \\
\hline & Chow + IMC & 0.032 & $22.67 \pm 1.03$ & $2.24 \pm 1.50$ & $16.06 \pm 3.11$ & & \\
\hline \multirow{2}{*}{ Plasma TMA $(\mu \mathrm{M})$} & Chow & 0.026 & $3.61 \pm 0.27$ & $0.62 \pm 0.36$ & $18.7 \pm 3.72$ & \multirow{2}{*}{$9.47 \mathrm{E}-10$} & \multirow{2}{*}{0.999} \\
\hline & Chow + IMC & 0.003 & $0.76 \pm 0.37$ & $0.62 \pm 0.24$ & $16.5 \pm 1.87$ & & \\
\hline \multirow{2}{*}{ Plasma TMAO $(\mu \mathrm{M})$} & Chow & 0.068 & $1.00 \pm 0.13$ & $0.16 \pm 0.13$ & $20.6 \pm 4.10$ & \multirow{2}{*}{$3.56 \mathrm{E}-09$} & \multirow{2}{*}{0.506} \\
\hline & Chow + IMC & 0.006 & $0.26 \pm 0.02$ & $0.05 \pm 0.02$ & $21.4 \pm 1.67$ & & \\
\hline \multirow{2}{*}{ Liver Foxo1 mRNA (AU) } & Chow & $5.42 \mathrm{E}-04$ & $1.22 \pm 0.12$ & $0.41 \pm 0.13$ & $16.82 \pm 1.71$ & \multirow{2}{*}{0.140} & \multirow{2}{*}{0.528} \\
\hline & Chow + IMC & 0.103 & $1.32 \pm 0.10$ & $0.24 \pm 0.20$ & $10.83 \pm 3.69$ & & \\
\hline \multirow{2}{*}{ Liver Chka mRNA (AU) } & Chow & 4.29E-05 & $0.78 \pm 0.06$ & $0.44 \pm 0.09$ & $21.39 \pm 0.31$ & \multirow{2}{*}{ 7.05E-08 } & \multirow{2}{*}{0.694} \\
\hline & Chow + IMC & $1.52 \mathrm{E}-04$ & $1.12 \pm 0.05$ & $0.38 \pm 0.10$ & $21.44 \pm 1.90$ & & \\
\hline \multirow{2}{*}{ Liver Pemt mRNA (AU) } & Chow & $5.46 \mathrm{E}-05$ & $1.02 \pm 0.06$ & $0.40 \pm 0.07$ & $16.9 \pm 0.77$ & \multirow{2}{*}{$6.34 \mathrm{E}-04$} & \multirow{2}{*}{0.186} \\
\hline & Chow + IMC & 0.403 & $1.26 \pm 0.11$ & $0.10 \pm 0.16$ & NA & & \\
\hline \multirow{2}{*}{ Muscle Taar5 mRNA (AU) } & Chow & 0.001 & $3.33 \pm 1.06$ & $2.90 \pm 1.26$ & $20.4 \pm 2.49$ & \multirow{2}{*}{$3.94 \mathrm{E}-03$} & \multirow{2}{*}{0.818} \\
\hline & Chow + IMC & 0.492 & $5.99 \pm 1.48$ & $2.02 \pm 3.51$ & NA & & \\
\hline \multirow{2}{*}{ Muscle Nr1d1 mRNA (AU) } & Chow & 0.001 & $0.47 \pm 0.08$ & $0.41 \pm 0.11$ & $4.26 \pm 1.14$ & \multirow{2}{*}{$1.88 \mathrm{E}-05$} & \multirow{2}{*}{0.079} \\
\hline & Chow + IMC & $1.01 \mathrm{E}-06$ & $0.89 \pm 0.13$ & $0.71 \pm 0.06$ & $4.63 \pm 0.52$ & & \\
\hline \multirow{2}{*}{ Muscle Pgc1 $\alpha$ mRNA (AU) } & Chow & 0.007 & $1.21 \pm 0.17$ & $0.22 \pm 0.17$ & $20.2 \pm 4.96$ & \multirow{2}{*}{$6.98 \mathrm{E}-04$} & \multirow{2}{*}{0.905} \\
\hline & Chow + IMC & 0.241 & $1.86 \pm 0.30$ & $0.28 \pm 0.35$ & NA & & \\
\hline
\end{tabular}




\section{Figure 4 - figure supplement 1}

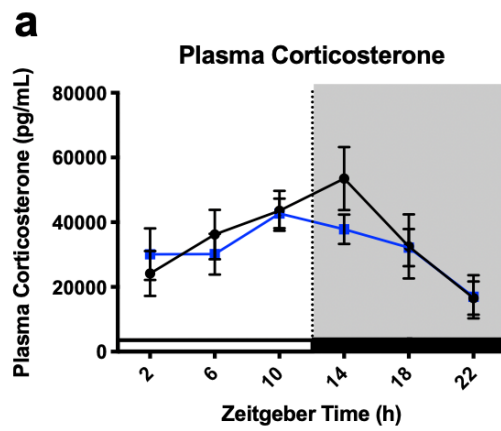

C

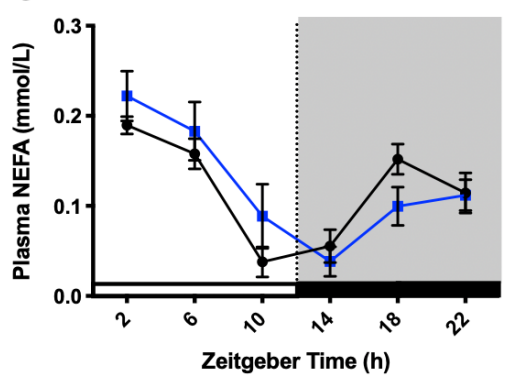

e Skeletal Muscle Taar5 mRNA levels

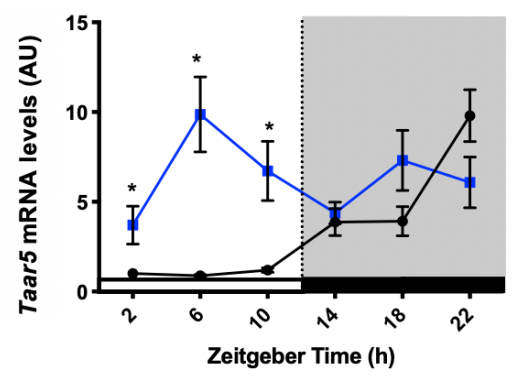

g Olfactory Bulb Taar5 mRNA levels

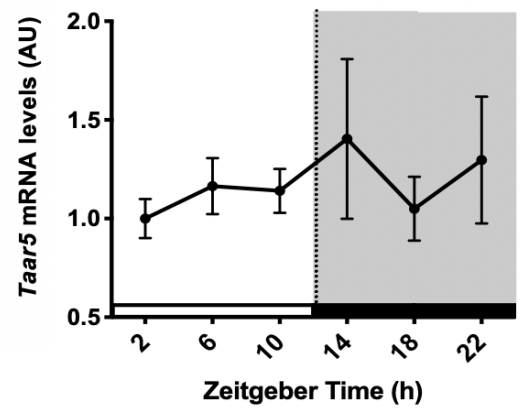

b

$\rightarrow$ Chow

$\rightarrow$ Chow + IMC

$\rightarrow$ Chow

$\rightarrow$ Chow + IMC

$\rightarrow$ Chow

- Chow + IMC

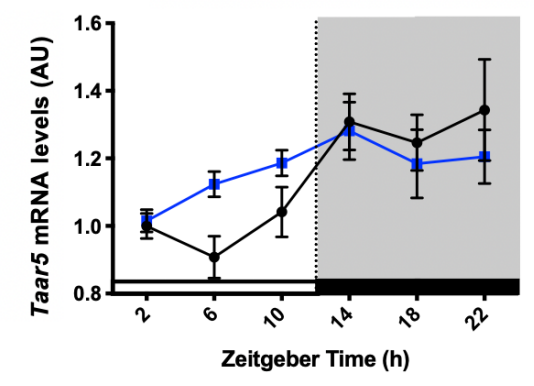

g

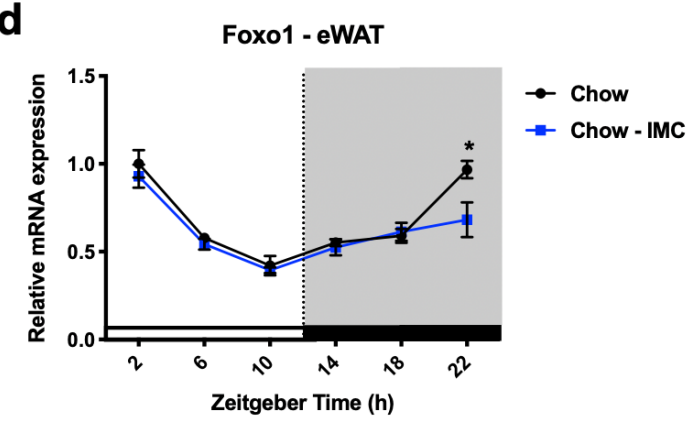

f

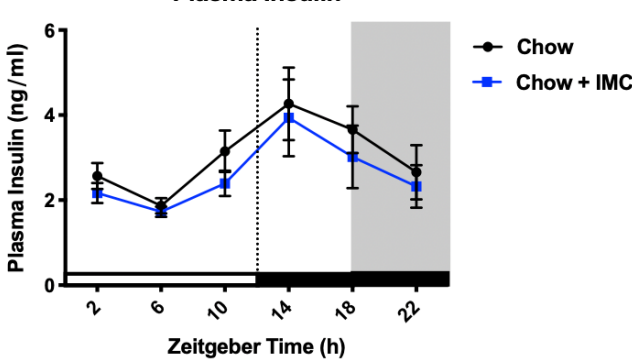

d

- Chow

-Chow + IMC

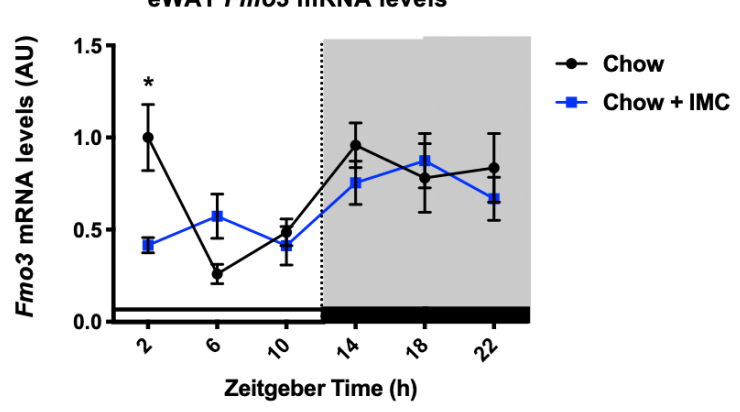

